\title{
FILLING A HOLE IN CYTOCHROME P450 BM3 IMPROVES SUBSTRATE BINDING AND CATALYTIC EFFICIENCY
}

\author{
Wei-Cheng Huang", Andrew C.G. Westlake『, Jean-Didier Maréchal, \\ M. Gordon Joyce, Peter C.E. Moody and Gordon C.K. Roberts* \\ Henry Wellcome Laboratories of Structural Biology, Department of Biochemistry, \\ University of Leicester, Leicester LE1 9HN, United Kingdom.
}

\footnotetext{
"These two authors contributed equally to this work

*Corresponding author: Professor Gordon Roberts, Department of Biochemistry, University of Leicester, Henry Wellcome Building, PO Box 138, Lancaster Road, Leicester LE1 9HN, United Kingdom. Telephone: +44(0)116 229 7100. Fax: +44(0)116 229 7053, E-mail: gcr@le.ac.uk
}

Running title: Substrate binding in cytochrome P450 mutants

Present addresses: Jean-Didier Maréchal: Departament de Química, Unitat de Química Física, Universitat Autònoma de Barcelona, Edifici C.n., 08193 Bellaterra, Spain; M. Gordon Joyce: Structural Immunology Section, Laboratory of Immunogenetics, National Institute of Allergy and Infectious Diseases, National Institutes of Health, 12441 Parklawn Drive, Rockville, MD 20852 USA Abbreviations used: P450s, cytochromes P450; P450BM3, cytochrome P450 BM3 from B. megaterium (CYP102A1); DTT, dthiothreitol; DMSO, dimethylsulfoxide; RMSD, root mean square deviation; TLC, thin layer chromatography. 


\section{Summary}

Cytochrome P450BM3 (CYP102A1) from Bacillus megaterium, a fatty acid hydroxylase, is a member of a very large superfamily of monooxygenase enzymes. The available crystal structures of the enzyme show non-productive binding of substrates with their $\omega$-end distant from the iron in a hydrophobic pocket at one side of the active site. We have constructed and characterised mutants in which this pocket is filled by large hydrophobic sidechains replacing alanine at position 82 . The mutants having phenylalanine or tryptophan at this position have very much $(\sim 800$-fold $)$ greater affinity for substrate, with a greater conversion of the haem iron to the high-spin state, and similarly increased catalytic efficiency. The enzyme as isolated contains bound palmitate, reflecting this much higher affinity. We have determined the crystal structure of the haem domain of the Ala82Phe mutant with bound palmitate; this shows that the substrate is binding differently from the wild-type enzyme but still distant from the haem iron. Detailed analysis of the structure indicates that the tighter binding in the mutant reflects a shift in the conformational equilibrium of the substrate-free enzyme towards the conformation seen in the substrate complex rather than differences in the enzyme-substrate interactions. On this basis, we outline a sequence of events for the initial stages of the catalytic cycle. The Ala82Phe and Ala82Trp mutants are also very much more effective catalysts of indole hydroxylation than the wild-type enzyme, suggesting that they will be valuable starting points for the design of mutants to catalyse synthetically useful hydroxylation reactions.

Keywords: cytochrome P450; substrate binding; crystal structure; indole; fatty acids 


\section{INTRODUCTION}

Cytochromes P450 are a superfamily, currently numbering more than 6000 , of haem-thiolate monooxygenase enzymes, found in almost all forms of life, which catalyse the activation of molecular oxygen ${ }^{1}$ and the addition of an atom of oxygen to their substrate. ${ }^{2}$ There is considerable sequence diversity within the superfamily; those members whose structures have been determined share a common overall fold, while differing markedly in their active site architecture, leading to very diverse substrate specificity. Members of the superfamily include both enzymes of high specificity involved in the biosynthesis of, for example, steroids (in mammals, insects, plants, fungi and bacteria ${ }^{3 ; 4 ; 5 ; 6}$ ), and polyketide antibiotics, ${ }^{4}$ and also 'de-toxifying' enzymes, including the well-known mammalian drug-metabolising P450s, ${ }^{3}$ which have a broad substrate specificity, allowing them to metabolise a very wide range of compounds.

The bacterial cytochromes P450 CYP101 (P450cam) from Pseudomonas putida and CYP102A1 (P450BM3) from Bacillus megaterium have been extensively studied structurally and mechanistically. ${ }^{7 ; 8 ;}$

${ }^{9}$ They have also been used as the starting point for the construction of mutants with altered specificity for use in chemical synthesis, where the ability of P450s to insert an oxygen atom into an unactivated $\mathrm{C}-\mathrm{H}$ bond has potentially valuable applications. $\mathrm{P} 450 \mathrm{BM} 3$, identified as a fatty acid hydroxylase, ${ }^{10 ; 11}$ is a 119kDa polypeptide which contains a P450 domain and a diflavin NADPH-cytochrome P450 reductase domain $^{11 ; 12}$ similar to that in the mammalian drug-metabolising mono-oxygenase system. Unlike most $\mathrm{P} 450 \mathrm{~s}$, therefore, which require additional electron transfer proteins, P450BM3 is catalytically selfsufficient. Perhaps because of this, P450BM3 also has the highest catalytic activity of any P450 monooxygenase identified to date.

P450BM3 catalyses the hydroxylation of C12-C16 saturated fatty acids at the $(\omega-1),(\omega-2)$ and $(\omega-$ 3) positions. ${ }^{10 ; 11}$ The crystal structure of the substrate-free form of the haem domain ${ }^{13}$ reveals a long hydrophobic active site channel extending from the protein surface, where there are charged residues suitable for binding a carboxylate group, to the haem iron - a structure entirely consistent with the observed fatty acid hydroxylase activity. The crystal contains two molecules in the asymmetric unit, one with an open substrate access channel and one where it is slightly more 'closed'; the two molecules have different intermolecular contacts in the crystal, and it seems likely that in solution there is a dynamic equilibrium between these and perhaps additional conformations of the access channel. ${ }^{13}$ In the crystal structures of the protein bound to the substrates palmitoleic acid ${ }^{14}$ and N-palmitoylglycine ${ }^{15}$ the protein is 
seen to have undergone a significant conformational change involving a displacement of the $\mathrm{F}$ and $\mathrm{G}$ helices. Studies of the A264E mutant have provided evidence to support the idea that these 'substratefree' and 'substrate-bound' conformations, which are quite distinct from the 'open' and 'closed' structures seen in the crystal structure of the free enzyme, coexist in equilibrium in solution in the absence of substrate. ${ }^{16}$ However, in none of the available structures of substrate complexes is the fatty acid bound with the $(\omega-1),(\omega-2)$ and/or $(\omega-3)$ carbons positioned close to the iron in a position for hydroxylation. Instead, the ' $\omega$ end' of the fatty acid becomes sequestered in a hydrophobic pocket between phenylalanines 81 and 87 , created by rotation of the aromatic ring of phenylalanine 87 by $\sim 90^{\circ}$ and a rearrangement of nearby side-chains (notably isoleucine 263 and leucine 437), with its terminal methyl group in contact with alanine 82 . In this position, the $\omega$ to $\omega-6$ carbons of the fatty acid are all between $7.5 \AA$ and $10 \AA$ from the iron centre, too distant for hydroxylation. (Binding of substrates remote from the haem has also been observed in crystal structures of a number of eukaryotic P450s, e.g. ${ }^{17 ; 18 ; 19}$.) All the available crystal structures of P450 BM3 thus represent inactive states of the enzyme-substrate complexes. This is in sharp contrast to P450cam, P450eryF and P450epoK, ${ }^{20 ; 21 ; 22 ; 23}$ where the natural substrates are bound such that the sites of hydroxylation are positioned within $5 \AA$ of the iron, and little or no rearrangement is observed relative to the substrate-free form. NMR relaxation experiments ${ }^{24}$ suggested that laurate and 12-bromo-laurate bind to $\mathrm{P} 450 \mathrm{BM} 3$ in solution at room temperature in a similar way to that seen for palmitate in the crystal structures. However, recent evidence from solid-state NMR for differences in the environment of some active site residues, including Phe81 and Phe87, in the $\mathrm{N}$-palmitoylglycine complex of $\mathrm{P} 450 \mathrm{BM} 3$ between room temperature and $-30^{\circ} \mathrm{C}$, led to the suggestion, ${ }^{25}$; ${ }^{26}$ supported by molecular dynamics simulations, ${ }^{27}$ that this may reflect an equilibrium between a low temperature binding mode which is seen in the crystal, and a different mode of binding at room temperature. It remains to be established whether the mode of binding seen in the fatty acid-P450BM3 complexes in the crystal is on the catalytic pathway, or whether it represents a 'dead-end' complex not normally populated under physiological conditions.

The existence of a hydrophobic pocket within the active site cavity, but relatively distant from the iron, also has implications for attempts to engineer the enzyme's activity towards novel target substrates. It seems likely that small hydrophobic molecules could preferentially bind in the pocket between 
phenylalanines 81 and 87 , resulting in non-productive complexes. There have been many reports of changing the substrate specificity of P450BM3 by substitution of phenylalanine 87 by a smaller residue

such as glycine, alanine or valine (e.g., $\left.{ }^{2} 28 ; 29 ; 30 ; 31 ; 32 ; 33 ; 34\right)$. Such substitutions would destroy the hydrophobic pocket, but would also increase the active site volume, which may result both in reduced coupling of NADPH consumption to product formation, due to less efficient exclusion of water from the active site, and in decreased regiospecificity of hydroxylation due to increased mobility of the substrate within the active site.

We have sought to investigate this issue by filling the hydrophobic pocket rather than destroying it. This we have done by substitution of alanine 82 by the larger hydrophobic residues isoleucine, phenylalanine and tryptophan. Examination of the structure suggests that these substitutions should exclude progressively larger volumes of the hydrophobic pocket and, in the case of the aromatic substitutions, perhaps fill it altogether. The mutants $\mathrm{A} 82 \mathrm{~F}$ and $\mathrm{A} 82 \mathrm{~W}$ are observed to bind fatty acids orders of magnitude more tightly compared to the wild type, and to show substantially increased catalytic efficiency. In addition, these mutants are found to be effective catalysts of the oxidation of indole, resulting in formation of indigo, suggesting that they may exhibit generally improved activity towards small molecules.

\section{RESULTS AND DISCUSSION}

As shown in Figure 1A, in the crystal structures of substrate complexes of P450 BM3 the fatty acid chain binds in a hydrophobic pocket between phenylalanines 81 and 87 , with the terminal methyl group in contact with Ala82. We have examined the substitution of Ala82 by a range of other residues. As shown in Figures 1B-D, this predicts that substitution by isoleucine, phenylalanine and tryptophan would be expected to fill progressively more of the pocket; the phenylalanine and tryptophan substitutions in particular would lead to a substantial predicted overlap with the end of the fatty acid chain, suggesting that they would prevent the binding of the fatty acid in the mode seen in the crystal structures. We therefore made and characterised the A82I, A82F and A82W mutants. 


\section{Fatty acid binding}

Unlike the wild-type enzyme and the A82I mutant, the A82F and A82W mutants as purified were in a predominantly high spin state, suggesting tight binding of a substrate-like molecule, presumably an endogenous fatty acid from the E. coli host cells. This could not be removed by extensive dialysis or by gel filtration. However, treatment of the purified protein with a small excess of NADPH, followed by buffer exchange using extensive ultrafiltration to remove reaction products, resulted in conversion to a predominantly low spin form. Final preparations of the A82F and A82W mutants contained $20 \%$ and $25 \%$ high spin haem respectively.

In line with these qualitative observations, marked differences in fatty acid binding were observed between the wild-type enzyme and the A82I mutant on the one hand, and the A82F and A82W mutants on the other (Figure 2, Table 1). Wild-type and A82I enzymes exhibited $\mathrm{K}_{\mathrm{d}}$ values of approximately 250 $\mu \mathrm{M}$ for laurate, with approximately $50 \%$ conversion to high-spin haem, whereas the $\mathrm{A} 82 \mathrm{~F}$ and $\mathrm{A} 82 \mathrm{~W}$ mutants bound this substrate 600 - to 800 -fold more tightly, with $\mathrm{K}_{\mathrm{d}}$ values of approximately $0.4 \mu \mathrm{M}$ and $>90 \%$ conversion to high spin. We attempted to study the kinetics of binding of laurate to the enzyme by stopped-flow methods, following the changes in the UV/visible absorption. However, in line with an earlier report on the wild-type enzyme, ${ }^{35}$ the observed rate was too fast $\left(>800 \mathrm{~s}^{-1}\right)$ for accurate determination by stopped-flow for either the wild-type or the A82F mutant.

In the case of palmitate, both wild-type and the mutant enzymes bound the substrate tightly and with substantial conversion to high spin haem (Table 1), but noticeable differences were again observed between wild-type and A82I on the one hand, and the A82F and A82W mutants on the other. Palmitate binding to $\mathrm{A} 82 \mathrm{~F}$ and $\mathrm{A} 82 \mathrm{~W}$ was too tight to determine by this method $\left(\mathrm{K}_{\mathrm{d}}<0.1 \mu \mathrm{M}\right)$ and was accompanied by complete conversion to high spin haem; in comparison wild-type and A82I gave between $85 \%$ and $90 \%$ high spin. The evidence that the endogenous fatty acid bound to the A82F enzyme as isolated is palmitate (see below) suggests that this substrate has a dissociation constant orders of magnitude below $0.1 \mu \mathrm{M}$, since it cannot be removed by dialysis. These data thus demonstrate that replacing Ala82 by larger, rigid sidechains leads to a marked increase in the affinity of the enzyme for fatty acids and also in the degree of conversion from low spin to high spin iron. By contrast, the substitution with the larger but more flexible isoleucine sidechain has essentially no effect. 


\section{Catalytic activity towards laurate}

The rates of NADPH consumption were measured for the mutant and wild-type enzymes with laurate as substrate; the catalytic activity against palmitate could not be estimated for either mutant or wild-type, since the rate was too fast to be measured in the standard assay and the protein concentration could not be lowered any further without dissociation of the catalytically functional dimer. ${ }^{36}$ The tighter binding of fatty acids to the $\mathrm{A} 82 \mathrm{~F}$ and $\mathrm{A} 82 \mathrm{~W}$ mutants was reflected in decreased $\mathrm{K}_{\mathrm{M}}$ values, while turnover rates were similar for all the mutants (Table 1). Assuming that $\mathrm{K}_{\mathrm{M}} \sim \mathrm{K}_{\mathrm{d}}$ for A82F and A82W as for wild-type and A82I, the combination of much tighter binding with unchanged turnover numbers leads to a much greater catalytic efficiency $\left(\mathrm{k}_{\text {cat }} / \mathrm{K}_{\mathrm{M}}\right)$ of the two mutants with aromatic side-chains at position 82 , estimated as $8 \times 10^{7} \mathrm{M}^{-1} \mathrm{~s}^{-1}(\mathrm{~A} 82 \mathrm{~F})$ and $1 \times 10^{8} \mathrm{M}^{-1} \mathrm{~s}^{-1}(\mathrm{~A} 82 \mathrm{~W})$ as compared to $1 \times 10^{5} \mathrm{M}^{-1} \mathrm{~s}^{-1}$ for the wild-type enzyme.

The products formed from the actions of the wild-type and mutant enzymes on laurate were identified by NMR spectroscopy; all of the mutants gave a mixture of $(\omega-1),(\omega-2)$ and $(\omega-3)$ products, with only small differences in product distribution. A82F and A82I appeared to favour $(\omega-2)$ hydroxylation compared to wild-type, whilst A82W gave a slightly higher proportion of ( $\omega-1)$ hydroxylated product (Figure 3).

The A82W mutant exhibited relatively poor coupling of NADPH consumption to product formation, as demonstrated by the significant amount of unmetabolised laurate remaining in the reaction mixture (Figure 3). Comparison of integrated peak areas allowed an estimate of approximately $45 \%$ coupling, whereas the other mutants gave much higher coupling efficiencies, estimated to be $>90 \%$ in all cases. A82W was also notably less stable than the other mutants, being inactivated after an average of $\sim 1000$ turnovers per enzyme molecule. In view of the relatively poor coupling, this mutant may be inactivated by generation of reactive oxygen species such as peroxide; addition of catalase to the reactions did indeed increase the lifetime of the protein, although it did not afford complete protection of activity, suggesting that that A82W may be inactivated by active oxygen species generated at the iron before they can diffuse out of the active site.

Both the A82F and the A82W mutants showed significantly higher rates of NADPH consumption in the absence of substrate than did the wild-type or A82I enzymes (wild-type $0.14 \mathrm{~s}^{-1}$, A82I $0.19 \mathrm{~s}^{-1}$, 
A82F $2.6 \mathrm{~s}^{-1}$, A82W $5.6 \mathrm{~s}^{-1}$ ); this is most likely to reflect the fact that, as noted above, these two mutants exist to a significant extent in the high-spin state in the absence of substrate.

\section{Crystal structure of the fatty acid complex of P450BM3 A82F}

In order to provide structural explanations for the marked differences in properties of the A82F and A82W mutants as compared to the wild-type enzyme, we determined the crystal structure of the haem domain of P450BM3 A82F. To date all crystallographic studies of P450BM3 have involved the haem domain of the enzyme rather than the intact enzyme, in which the flexibility of the two domains may inhibit crystallisation. We therefore expressed and purified the haem domain of the A82F mutant. As with the full-length protein, the A82F haem domain was isolated in a high spin form, suggesting again that a substrate-like molecule was bound; this could not be removed either by gel filtration or extensive dialysis (nor, in the absence of the reductase domain, by NADPH-supported catalytic turnover). Its identity was established by extracting a protein sample with dichloromethane, and analysis of the resultant solution by liquid chromatography-mass spectroscopy. The dominant component was identified as palmitic acid, with $\mathrm{m} / \mathrm{z}=255$ and an identical retention time to an authentic standard; spiking the sample with authentic standard confirmed the identification. The presence of this fatty acid in the active site of $\mathrm{A} 82 \mathrm{~F}$ is unsurprising; as described above, palmitate binds extremely tightly to the full-length protein, and it has been identified as one of the three major fatty acids in the E. coli host cells. ${ }^{37}$

Since the palmitate could not be removed, we crystallized the substrate complex of the haem domain using conditions similar to those previously reported. ${ }^{16}$ Crystals were obtained in the $\mathrm{P} 2{ }_{1} 2_{1} 2_{1}$ space group, and diffracted to $2.8 \AA \AA$ resolution; in contrast to previous P450BM3 crystals, six chains were identified in the asymmetric unit. The structure was solved by molecular replacement, using the wildtype N-palmitoylglycine-bound structure (PDB 1JPZ) as a search model. Strong electron density was observed in the active site indicating the presence of bound fatty acid, and the structure was therefore refined with palmitate modelled in this position; the extent of the electron density was entirely consistent with the identification of the bound fatty acid as palmitate. The final model shows well-defined electron density for both the substrate and active site residues, including the phenylalanine side-chain at position 82. 
As can be seen in Figure 4A, the structure of the A82F mutant is very similar to that of the wildtype haem domain complexed with N-palmitoylglycine (PDB 1JPZ), and also to the palmitoleic acid complex (PDB 1FAG). The six chains in the unit cell all adopt essentially the same conformation, with pairwise RMSD values between 0.49 and $0.83 \AA$; the differences between them are confined mainly to loop regions and surface side chains, particularly to regions which are known to move on substrate binding, including the A helix, the $\beta 1$ region and the F-G loop. There are however some noticeable differences in the binding of the substrate between the different chains in the crystal; these are discussed below.

The replacement of the small aliphatic side chain of A82 with the bulky aromatic side chain of phenylalanine in the mutant is readily accommodated with minimal changes to the protein structure compared to the two reported substrate complexes of the wild-type enzyme (RMSD $<0.74 \AA$; Figure $4 A$ ). The ring of Phe 82 fits between Phe81 and Phe87, and as predicted fills the hydrophobic pocket into which the fatty acid terminus binds in the wild-type structures (Figure 4B). This changes the shape of the substrate binding channel and reduces its overall length, forcing the substrate to adopt a different binding position, with the carboxylate group closer to the protein surface (Figure 4C). This apparently results in disruption of a hydrogen bond normally formed between the substrate carboxylate group and tyrosine 51 . Perhaps as a result of this, there are distinct differences in the binding position of the substrate between the six chains in the unit cell. This is in sharp contrast to the wild type structures, where the four chains in $1 \mathrm{FAG}$ and the two chains in $1 \mathrm{JPZ}$ each exhibit almost identical substrate binding modes. In A82F, the greatest difference in substrate binding position is between chains $\mathrm{E}$ and $\mathrm{F}$, with a $2.5 \AA$ shift in the location of the carboxylate group between these two; the carboxylate is also between 2 and $4 \AA$ closer to the surface than is that of palmitoleic acid in the wild type complex. The considerable conformational flexibility of the sidechain of arginine 47 appears to help compensate for the repositioning of the carboxylate group between the various structures.

The 'filling in' of the hydrophobic pocket in the substrate binding channel by the A82F mutation results in a significant displacement of the fatty acid $(\omega-1)$ to $(\omega-3)$ carbons, which are the sites of metabolism, from their positions in the wild-type structures. The terminal methyl group of the substrate

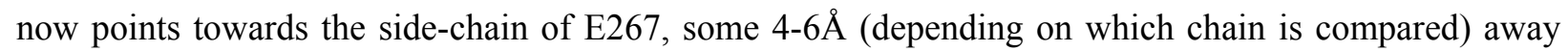
from its location adjacent to A82 in the wild type structure (Figure 4C). The key amino-acid residues in 
the binding site adopt essentially identical positions to those in the N-palmitoylglycine complex of the wild-type enzyme (Figure 4C). Notwithstanding the fact that the binding position of the substrate has changed, there is virtually no change in the regiospecificity of hydroxylation.

In the structure of the complex of the wild-type enzyme with N-palmitoyl-glycine $\left(1 \mathrm{JPZ}^{15}\right)$, there is no water molecule coordinated to the iron (consistent with a high-spin complex), but there is an ordered water molecule positioned close to the iron and hydrogen bonded to A264 and T268. In the palmitoleic acid complex $\left(1 \mathrm{FAG}^{14}\right)$, there are no water molecules in the PDB file, but a similarly located water molecule is seen in the electron density map for two of the four chains. This water molecule was identified by Haines et al. ${ }^{15}$ as potentially crucial to the mechanism of proton transfer in P450BM3. However, in the crystal structure of the palmitate complex of the A82F mutant there is electron density corresponding to this water molecule only in one of the six chains (chain D) (Figure 4B and Supplementary Material). It is not clear why this should be so, since there is space for this water in the structure and the potentially hydrogen bonding residues are in the same place; it may be that the altered position of the terminal methyl group of the substrate makes this location more hydrophobic and thus water binding weaker. In any case, the absence of this water in the structure of the A82F mutant, which has a greater catalytic efficiency than the wild-type enzyme, raises questions about the mechanistic importance of a tightly bound water molecule near the iron. There is no doubt that it is essential for a water molecule to have access to this region of the active site close to the haem, and probably to be oriented by hydrogen-bonding to $\mathrm{T} 268^{38}$, in order to participate in essential proton transfers, but it may not be necessary for it to be tightly bound in the oxidised form of the complex.

All available crystal structures of substrate complexes of P450BM3 clearly represent inactive states of the enzyme-substrate complex. Unlike in other structures such as P450cam where substrate is bound with the position of metabolism close to the iron centre, ${ }^{20 ; 21 ; 39}$ in the complexes of wild-type or the A264E mutant of P450BM3 with palmitoleic acid ${ }^{14 ; 16}$ or of wild-type enzyme with N-palmitoylglycine $^{15}$ the fatty acid is bound distantly from the iron. Similarly in the structure of the palmitate complex of the A $82 \mathrm{~F}$ mutant the fatty acid binds towards the 'roof' of the active site pocket (Figure 4B,C), and the distances from the iron centre to the $(\omega-1)-(\omega-3)$ carbons, while somewhat shorter than in the wild-type complexes, are still between 7 and $9 \AA$, suggesting that, as in the wild type, the crystal structure obtained represents an unproductive mode of substrate binding. For none of the structures of 
P450BM3 is there unambiguous evidence that the crystals are catalytically active; widespread attempts to reduce the enzyme in the crystal have met with failure due to crystal cracking. However, since very similar structures of the enzyme-substrate complex are obtained for different substrates, for wild-type and mutant enzyme and in different space groups, it seems likely that these structures do represent relevant complexes. Thus it appears that, at some point in the catalytic cycle after the initial substrate binding, both wild-type and A82F P450BM3 must rearrange to conformations where the fatty acid substrate binds closer to the iron. Indeed previous work in our laboratory using paramagnetic relaxation experiments has suggested that substrates may move by as much as $6 \AA$ on reduction of the iron centre in the wild type protein. ${ }^{40}$ The very similar product distributions in wild-type and A82F suggest that in this 'rearranged' conformation the substrate has a similar orientation in both wild-type and mutant complexes.

MacDermott et al. ${ }^{25 ; 26 ; 27}$ have linked this requirement for a movement of the substrate into a 'catalytic' position to the equilibrium between low-spin and high-spin states of the complex, corresponding to six-coordinate (with an axial water ligand) and five-coordinate states of the haem iron. Noting the fact that the low-spin - high-spin equilibrium is clearly temperature dependent in the wildtype enzyme, ${ }^{26}$ they have suggested that the crystal structure of the N-palmitoyl-glycine complex, in which the substrate is bound in a 'distantly bound' position, corresponds to a low-spin state. As discussed above, in this complex there is a water molecule very close to the iron; ${ }^{15}$ however the distance and angle between the water and the iron, along with the lack of any bonding electron density between them, are more consistent with the original assignment of the crystal structure as a high spin complex. MacDermott et al. $^{26 ; 27}$ suggest that at room temperature, where the complex is in the high-spin state and where catalytic activity is observed, the substrate has moved close to the iron. It is clear from the available structures that the aromatic ring of phenylalanine 87 appears to form a 'barrier' between the substrate in its 'distantly bound' position and the haem iron, and they propose that rotation of this ring is a key feature of the room temperature high-spin complex. ${ }^{26 ; 27}$

In the light of this evidence for a temperature dependence of the low-spin - high-spin equilibrium and perhaps of the mode of substrate binding, we examined the UV/visible spectra of the palmitate complexes of the wild-type and mutant enzymes as a function of temperature between $4^{\circ} \mathrm{C}$ and $30^{\circ} \mathrm{C}$ (Figure 5). The wild-type enzyme and the A82I mutant showed significant shifts to low-spin as the temperature was decreased, reaching an estimated $50 \%$ low-spin at $4^{\circ} \mathrm{C}$ for wild-type and $70 \%$ low-spin 
for A82I; however, in marked contrast there was no significant temperature dependence of the spectra for the $\mathrm{A} 82 \mathrm{~F}$ or $\mathrm{A} 82 \mathrm{~W}$ mutants, which remained completely high-spin at all temperatures examined. Taken together with the absence of a water molecule near the haem iron in the crystal structure, this strongly suggests that the palmitate complex of the A82F mutant is high-spin both in the crystal and in solution, and hence that the movement of the substrate into the 'catalytic' position is not coupled to the spin-state change; rather, a complete spin-state change can occur with the substrate in the 'distantly bound' position.

\section{Structural origin of the tighter fatty acid binding}

Experimentally we observe that fatty acids bind $\sim 1000$-fold more tightly to A $82 \mathrm{~F}$ than to the wild-type enzyme. However, the crystal structure of the palmitate complex offers no clear-cut explanation for this. Indeed the position of the fatty acid appears less ordered than in the wild-type, presumably due to disruption of the hydrogen bond normally formed between the carboxylate and tyrosine 51 , and this would be expected to result in weaker binding. Substitution of alanine 82 with the larger phenylalanine residue is likely to exclude water more efficiently from the complex, resulting in somewhat tighter binding due to hydrophobic effects, but it seems unlikely that this effect would be sufficient to explain the magnitude of the observed decrease in $\mathrm{K}_{\mathrm{d}}$; experiments using camphor derivatives designed to fill in 'holes' in the protein-substrate complex of P450cam resulted in much more modest increases in binding affinity. ${ }^{41}$

Since comparison of the substrate-bound structures provides no clear-cut explanation for the tighter binding to the $\mathrm{A} 82 \mathrm{~F}$ mutant, we need to consider in more detail the complete substrate binding process, and in particular the conformational change which occurs on substrate binding to wild-type P450BM3 ${ }^{14 ; 15}$. Examination of the crystal structure of the substrate-free wild-type enzyme shows that alanine 82 is surrounded closely by phenylalanines 81 and 87 and isoleucine 263 , such that there appears to be insufficient space to tolerate the substitution to phenylalanine (Figure 6). In the structure of the substrate complexes of the wild-type enzyme, the alkyl chain of the fatty acid displaces the side chains of isoleucine 263 and leucine 437, rotating them towards the F and $\mathrm{G}$ helices, and directly resulting in the repacking and displacement of this structural unit; the $\mathrm{F}$ helix is displaced about half a turn along its length, and by about two-thirds of its width laterally, between the two conformations. As noted above, 
the structure of the palmitate complex of the A $82 \mathrm{~F}$ mutant is very similar to that of the substrate complexes of the wild-type enzyme, and this is specifically true of this region of the structure and of Ile263. Examination of these structures suggests that the bulky side-chain of phenylalanine 82 itself would induce the same conformational change in isoleucine 263 (and hence in the F and G helices) in the absence of the substrate (Figure 6). The structural evidence thus indicates that, in the absence of substrate, P450BM3 A82F would adopt a conformation closely resembling the substrate-bound form (as observed for the A264E mutant ${ }^{16}$ ). The substantial increase in binding affinity for fatty acids observed as a result of the substitution of Ala82 by phenylalanine would thus arise not from any change in interactions between the substrate and the enzyme as a result of the mutation, but rather from a shift in the conformational equilibrium in the free enzyme. By contrast, examination of the structure indicates that substitution of alanine 82 by isoleucine can be accommodated in the substrate-free conformation and would not perturb the conformational equilibrium; this would be consistent with the observation that the A82I mutant resembles the wild-type in many of its properties.

\section{The substrate binding process}

Combining the information from the present work with that already available from structural, spectroscopic and simulation studies allows us to postulate a sequence of events for the early stages of the catalytic cycle of P450BM3. First, we suppose that the free enzyme exists in an equilibrium between two conformations, corresponding to the 'substrate-free' and 'substrate-bound' conformations. The substrate binds preferentially to the 'substrate-bound' conformation; the data for the A82F and A82W mutants suggest that this preference must be several hundred-fold, although there is insufficient information available to provide a precise value. In this initial complex, the substrate is 'distantly bound', with the $(\omega-1)$ to $(\omega-3)$ carbons $7-9 \AA$ from the iron. In spite of this, the spin state equilibrium is shifted over towards the high-spin state - almost completely in the case of palmitate binding. This spin state shift is attributed to displacement of the water molecule from the sixth coordination position of the iron. The substrate itself is clearly too far from the iron to displace the water directly; however, the bound substrate makes contact with the sidechain of Phe87, leading to a reorientation of this sidechain ${ }^{14 ; 15}$ which would in turn displace the water. There is space for the displaced water to remain within the active site, where it is likely to play a role in proton transfers, but from the structure of the $\mathrm{A} 82 \mathrm{~F}$ complex it appears that tight 
binding of the displaced water is not essential. If the low-spin - high-spin transition arises from the rotation of Phe87, what is it that prevents this from occurring in the absence of substrate? In the 'substrate-free' conformation of the enzyme, Leu437 extends into the active site and approaches Phe87 quite closely; its two methyl groups appear to lock the ring of Phe87 in the 'vertical' position, preventing it from displacing water, and keeping the iron low-spin. Substrate binding and the conversion to the 'substrate bound' conformation displaces Leu437 significantly away from Phe87, such that the ring can now rotate and displace the water ligand from the iron. In the $\mathrm{A} 82 \mathrm{~F}$ and $\mathrm{A} 82 \mathrm{~W}$ mutants, the substitution itself leads to displacement of Ile263 and hence Leu437, and allows rotation of Phe87; as a result, for both these mutants there is a significantly larger proportion of high-spin iron in the absence of substrate $(20-25 \%)$, together with a greater 'leak rate' of NADPH consumption in the absence of substrate.

The next step in the catalytic cycle is the essential movement of the substrate into a position closer to the iron, appropriate for hydroxylation, and again Phe87 appears to play a key role. Examination of the structure suggests that this residue forms a 'barrier' to this movement, and support for this comes from studies of isotope effects on the hydroxylation of small substrates by wild-type P450BM3 and the F87A mutant. ${ }^{42}$ Molecular dynamics simulations provide evidence for the coupling of the movement of the substrate and the movement of the sidechain of Phe $87 .{ }^{27}$ This central role for Phe 87 is consistent with a

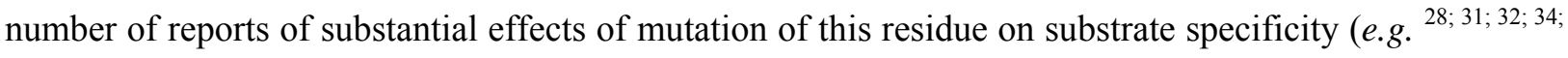
${ }^{43}$ ). Further work aimed at providing support for this postulated sequence of events is in progress.

\section{Oxidation of indole by A82 mutants}

Unlike the wild type enzyme or the A82I mutant, cells expressing P450BM3 A82F or P450BM3 A82W were observed to produce an insoluble blue dye during normal growth. This has been previously observed with certain human P450 isoforms, notably CYP2A6 $6^{44 ; 45}$ and with the F87V mutant of $\mathrm{P} 450 \mathrm{BM} 3{ }^{46}$, and attributed to the formation of indigo by oxidation of indole either present in the growth medium or endogenous to the E. coli host cells. The simple colorimetric nature of this phenomenon has also led to its use in screening libraries of random mutants to identify variants of P450BM3 and CYP2A6

with enhanced indole hydroxylation activity. ${ }^{46 ;}{ }^{47}$ Wild-type P450BM3 has been reported to produce detectable amounts of indigo only at high $\mathrm{pH}$ in a reaction driven by cumene hydroperoxide rather than 
$\mathrm{NADPH}^{48}$. We therefore investigated further the interaction of the purified A82 mutant enzymes with indole.

The binding of indole to wild-type and mutant P450BM3 gave type I spectral changes in the haem Sorêt UV/visible absorbance, with a decrease in absorbance at $420 \mathrm{~nm}$ and an increase at $390 \mathrm{~nm}$, indicative of displacement of the axial water ligand from the haem iron and conversion to the high spin state (Figure 7). For the wild-type enzyme and the A82I mutant, plots of $\Delta\left(\mathrm{A}_{390}-\mathrm{A}_{420}\right)$ vs. indole concentration demonstrated weak binding, with estimated apparent dissociation constants of $\sim 10 \mathrm{mM}$ or greater; accurate values could not be obtained due to the limited solubility of indole. By contrast, the A82F and A82W mutants, which also gave typical type I substrate binding spectra at indole concentrations $\leq 10 \mathrm{mM}$, clearly bind indole much more tightly. Data in the concentration range up to 8 $\mathrm{mM}$ indole were best fitted by a two-site binding model, with apparent $\mathrm{K}_{\mathrm{d}}$ values for the tight binding site of $\sim 0.1 \mu \mathrm{M}$, similar to the concentration of protein used in the experiment, and $\mathrm{K}_{\mathrm{d}}$ values for the second site $\sim 1000$-fold higher (Table 2). Even the weaker site shows indole binding at least 35 - to 65 -fold tighter than that observed for wild-type enzyme. The two sites contributed approximately equally to the final overall absorbance change, and the magnitude of this change showed that the total spin-state changes on indole binding were substantial, approximately $80 \%$ for $\mathrm{A} 82 \mathrm{~W}$ and $95 \%$ for $\mathrm{A} 82 \mathrm{~F}$, indicating efficient displacement of the axial water ligand from the haem iron. On addition of high concentrations $(\sim 18 \mathrm{mM})$ of indole to the $\mathrm{A} 82 \mathrm{~F}$ or $\mathrm{A} 82 \mathrm{~W}$ mutants a further spectral change was observed (Figure 7), with a shift in the Sorêt peak to $424 \mathrm{~nm}$ to give a Type II binding spectrum, typical of inhibitor complexes involving direct nitrogen coordination to the haem iron, suggesting the weak binding directly to the haem iron. The substitution of alanine 82 by larger, rigid sidechains in the A82F and A82W mutants thus leads to a very marked increase in affinity of the enzyme for indole. Studies of indole turnover demonstrate that this is accompanied by a substantial increase in catalytic efficiency.

Indole hydroxylation by P450 enzymes generally gives rise to a complex mixture of soluble and insoluble products ${ }^{45 ; 46}$ (see Supplementary Material). Initial hydroxylation may take place at either the 2position to give oxindole, or at the 3-position to give the rather unstable indoxyl, which dimerises through a non-enzymatic pathway to give the insoluble dye indigo. In addition, indoxyl may be further oxidised, most probably non-enzymatically, to isatin, which can then form a heterodimer with indoxyl to generate the insoluble dye indirubin. The soluble and insoluble products from the action of wild-type and 
mutant P450BM3 on indole were separated by centrifugation. The soluble compounds were characterised by HPLC, while the water-insoluble dyes were redissolved in DMSO and analysed by TLC, optical spectroscopy and ${ }^{1} \mathrm{H}-\mathrm{NMR}$; details of the analyses are given in the Supplementary Information. The predominant soluble product was oxindole, with trace quantities of isatin, while the major waterinsoluble product was indigo, with only a small amount of indirubin.

For wild-type enzyme and the A82I mutant, plots of the rate of NADPH consumption vs. indole concentration did not show complete saturation by $18 \mathrm{mM}$ indole, consistent with the low affinity for indole indicated by the optical titration experiments. The data were best fitted by the Hill equation (modified to account for the non-zero rate of NADPH consumption in the absence of substrate), leading to estimated Hill coefficients of 1.7 in both cases. The kinetic parameters for the wild-type enzyme (Table 2) are in agreement with those published previously. ${ }^{48}$ For the A82F and A82W mutants an increase in NADPH consumption rate with increasing indole concentration was observed at concentrations up to approximately $8 \mathrm{mM}$, above which marked inhibition occurred. This is consistent with the optical binding studies, which demonstrate formation of a type II complex at these concentrations, typical of inhibitor binding. Estimating the kinetic parameters from the data at indole concentrations below $8 \mathrm{mM}$, it is clear that the $\mathrm{K}_{\mathrm{M}}$ values are lower (by a factor of 4.7 for $\mathrm{A} 82 \mathrm{~F}$ and 81 for $\mathrm{A} 82 \mathrm{~W}$ ) and the $\mathrm{V}_{\max }$ values higher, by a factor of 6 , for $\mathrm{A} 82 \mathrm{~F}$ and $\mathrm{A} 82 \mathrm{~W}$ than for the wild-type; in addition, for these mutants the Hill coefficient is not significantly different from one (Table 2). Little increase in the NADPH consumption rate over that observed in the absence of substrate was observed at low indole concentrations, suggesting at most a modest contribution to the turnover rates by the very tight indole binding site observed by optical titration.

The coupling of product formation to NADPH consumption in the hydroxylation of indole was determined by measuring the formation of oxindole and indigo in reaction mixtures containing $8 \mathrm{mM}$ indole and $200 \mu \mathrm{M}$ NADPH; by ignoring the small amounts of isatin and indirubin formed, this will slightly underestimate the degree of coupling. As shown in Table 4, very little product was generated by wild-type enzyme or the A82I mutant, and the coupling was very poor. The substitution of Ala82 by phenylalanine or tryptophan, on the other hand, led to marked increases in the coupling of product formation to NADPH consumption, and to a 170 -fold increase in the rate of product formation. Taken together with the $\geq 25$-fold increase in $\mathrm{k}_{\text {cat }} / \mathrm{K}_{\mathrm{M}}$ values estimated from the measurements of NADPH 
turnover, it is clear that the $\mathrm{A} 82 \mathrm{~F}$ and $\mathrm{A} 82 \mathrm{~W}$ substitutions lead to a substantial increase in the efficiency of indole hydroxylation by the enzyme.

\section{CONCLUSIONS}

Cytochrome P450BM3 is well-known to catalyse the hydroxylation of C12-C16 saturated fatty acids at the $(\omega-1),(\omega-2)$ and $(\omega-3)$ positions. ${ }^{10 ; 11}$ However, one of the continuing puzzles with regard to this enzyme is the fact that in none of the available structures of substrate complexes is the fatty acid bound with these carbons positioned close enough to the iron for hydroxylation. Instead, the ' $\omega$ end' of the fatty acid becomes sequestered in a hydrophobic pocket between phenylalanines 81 and 87 , with the carbons

which are hydroxylated all between $7.5 \AA$ and $10 \AA$ from the iron centre, clearly too distant for hydroxylation. This is in contrast to enzymes such as $\mathrm{P} 450$ cam and $\mathrm{P} 450 \mathrm{eryF},{ }^{20 ; 21 ; 22}$ where the natural substrates are bound in such a way that the sites of hydroxylation are positioned within $5 \AA$ of the iron. In an attempt to understand the significance of this hydrophobic pocket in substrate binding, we have made a series of mutants at alanine 82 in which the pocket is predicted to be filled by hydrophobic sidechains in such a way as to prevent the binding of fatty acids in the way seen in structures of the wild-type enzyme. The structure of the A82F mutant in its complex with palmitate showed that the pocket had indeed been filled by the sidechain of Phe82 and the mode of binding of the fatty acid had indeed changed. However, rather than finding that the $\omega$ end of the fatty acid was now binding closer to the iron, we observed a shift in the position of the fatty acid towards the surface of the protein so that the $(\omega-1)$, $(\omega-2)$ and $(\omega-3)$ carbons remained 7-9 $\AA$ from the iron. This clearly indicates that this 'distant' binding position does not result simply from the availability of the hydrophobic pocket between Phe 81 and Phe87.

Nonetheless, the $\mathrm{A} 82 \mathrm{~F}$ and $\mathrm{A} 82 \mathrm{~W}$ mutants, in which this pocket has been filled with large rigid sidechains, showed a remarkable increase in affinity for fatty acids - to the extent that endogenous palmitate could not be removed by extensive dialysis - and in catalytic efficiency. It is unusual for a simple single-site substitution to lead to such a marked ( $~ 800$-fold) increase in substrate affinity, but detailed comparison of the mutant and wild-type complexes did not reveal any differences in the enzyme- 
substrate interactions which are likely to account for this. The most likely explanation is that the substitution of the large sidechains at position 82 favours the 'substrate-bound' conformation, seen in the complexes with palmitoleate and N-palmitoyl-glycine and in the substrate-free A264E mutant, over the 'substrate-free' conformation, due to interactions of Phe82 or Trp82 with Ile263, leading in turn to movements of the $\mathrm{F}$ and $\mathrm{G}$ helices. As discussed by Joyce et al., ${ }^{16}$ the free enzyme may exist in an equilibrium between these two conformations. By changing the position of this equilibrium in the absence of substrate, the mutations will decrease the proportion of the binding energy used up in shifting the equilibrium on substrate binding, leading to a net increase in affinity.

The observation of the formation of a blue pigment by cells expressing the A82F or A82W mutants of P450BM3 was the first indication that these mutants might be able efficiently to hydroxylate indole, with the formation of indigo, and this was unambiguously confirmed by studies of the isolated enzymes. The data suggest that, whilst both wild-type and A82I can bind indole, albeit weakly, it adopts a predominantly unproductive binding mode in the active sites of these proteins, since the amount of product formed is so low. It is possible that a significant proportion of the indole bound to these enzymes is located in the hydrophobic pocket bordered by Phe81, Phe87 and residue 82 . In the mutants in which this pocket is filled by a larger sidechain at position 82 , the efficiency of hydroxylation of indole is markedly increased, due both to increased $\mathrm{k}_{\text {cat }} / \mathrm{K}_{\mathrm{M}}$ values and to increased coupling between NADPH consumption and product formation. The evidence suggests that indole binding to P450BM3 is complicated, involving the binding of several indole molecules in catalytically productive, nonproductive and inhibitory positions; there is other evidence for the simultaneous binding of more than one substrate molecule to P450BM3 and its mutants. ${ }^{49 ; 50}$ Notwithstanding these complications, the A82F and $\mathrm{A} 82 \mathrm{~W}$ mutants are clearly much more effective catalysts of indole hydroxylation than is the wildtype enzyme. There are two possible contributions to this improved catalytic efficiency. First, the substitution will lead to the removal of the potential non-productive binding site in the hydrophobic pocket; simple docking calculations (Figure 8) do indeed suggest that the preferred binding site for indole in the wild-type enzyme is the hydrophobic pocket, while in the A82F mutant it prefers to bind much closer to the haem, with the 2- and 3-positions closest to the iron, in position for hydroxylation. Secondly, if, as suggested above, the mutation leads to a shift in the conformational equilibrium of the enzyme, this will increase the affinity for indole and in addition, with its effect on the environment of 
Phe87, will promote conversion to the high-spin state and hence the rate of electron transfer. Whichever is the more important contribution, the present results suggest that these mutants are not only very efficient fatty acid hydroxylases but also efficient hydroxylases of small hydrophobic molecules and hence may be useful biocatalysts in organic synthesis ${ }^{51 ; 52}$ and perhaps in the biodegradation of polycyclic aromatic hydrocarbons. ${ }^{28 ; 53}$

\section{MATERIALS AND METHODS}

\section{Materials}

The QuikChange XL mutagenesis kit was obtained from Stratagene, UK, and oligonucleotide primers from the Protein and Nucleic Acid Chemistry Laboratory, University of Leicester, UK. Restriction enzymes were obtained from New England Biolabs. Chromatography columns and media from were obtained from Amersham Biosciences, Complete ${ }^{\mathrm{TM}}$ Protease inhibitors from Roche and indirubin from Biomol International LP, UK. All other chemicals, of analytical grade or higher, were from Sigma Aldrich UK Ltd.

\section{Site-directed Mutagenesis}

Wild-type and mutant proteins were expressed using the plasmid pGLWBM3, encoding full-length P450BM3, which was a kind gift of Professor L.-L. Wong, University of Oxford, UK. The plasmid was modified to incorporate a number of silent restriction sites to facilitate easy recombination of mutants in regions of the gene corresponding to the various substrate recognition sites, and to allow simple excision of the region encoding the reductase domain, thus providing a straightforward route to expression of the haem domain of mutants of interest for structural studies (full details are given in the Supporting Information). Mutagenesis was carried out using the Stratagene QuikChange XL kit according to the manufacturer's instructions. Primers used were as follows (mismatches are underlined):

\footnotetext{
A82I F : 5' -GCTTAAATTTGTACGTGATTTTATCGGAGACGGGTTATTTACAAGC - 3'

A82I R: 5' - GCTTGTAAATAACCCGTCTCCGATAAAATCACGTACAAATTTAAGC - 3'

A82F F : 5' - GCTTAAATTTGTACGTGATTTTTTCGGAGACGGGTTATTTACAAGC - 3'

A82F R: 5' - GCTTGTAAATAACCCGTCTCCGAAAAAATCACGTACAAATTTAAGC - 3'
} 
A82W F : 5' -GCTTAAATTTGTACGTGATTTTTGGGGAGACGGGTTATTTACAAGC - 3'

A82W R: 5' -GCTTGTAAATAACCCGTCTCCCCAAAAATCACGTACAAATTTAAGC - $3^{\prime}$

All mutant genes were fully sequenced to confirm the absence of any undesired mutations.

\section{Protein expression and purification}

Protein was expressed and purified using a modification of methods previously described, ${ }^{28}$ the fulllength protein and the haem domain were purified in essentially the same way. Briefly, Escherichia coli JM109 cells harbouring pGLWBM3 were grown in $2 \times$ YT medium containing $100 \mu \mathrm{g} / \mathrm{ml}$ ampicillin at $30^{\circ} \mathrm{C}$ for $6-8$ hours to mid-log phase. The growth medium was supplemented by the addition of $0.5 \mathrm{mM}$ $\delta$-aminolaevulinic acid, $25 \mathrm{mM}$ ferric citrate and $1 \mathrm{ml} /$ litre of a saturated solution of riboflavin, and incubation was continued for 12-16 hours. Cells were harvested by centrifugation, resuspended in Buffer A (50 mM potassium phosphate, $1 \mathrm{mM}$ EDTA, $1 \mathrm{mM}$ benzamidine, $1 \mathrm{mM}$ DTT, $\mathrm{pH}$ 7.5) supplemented with Complete ${ }^{\mathrm{TM}}$ Protease inhibitors, and stored at $-20^{\circ} \mathrm{C}$ until processed. All of the A82 mutants expressed well in E. coli, producing levels of polypeptide similar to those seen for the wild-type enzyme. Cells were lysed by sonication, and the soluble fraction was loaded onto a DEAE-Sepharose Fast-Flow column pre-equilibrated with Buffer A. The protein was eluted using a gradient of 0-0.5 M KCl in buffer A, and fractions exhibiting the highest haem content pooled, and concentrated and desalted by ultrafiltration. This was then loaded onto a HiLoad Q-Sepharose 26/10 HP column pre-equilibrated with Buffer A, and eluted with a gradient of 0.2-0.5 M KCl in buffer A. Again fractions with the highest haem content were pooled, and concentrated and desalted by ultrafiltration. Finally, to remove any bound substrate-like molecules from the full-length enzyme, the protein was treated with 5 molar equivalents of NADPH for 5 minutes at room temperature, and any products removed by extensive ultrafiltration prior to storage at $-20{ }^{\circ} \mathrm{C}$. Purification led to protein of $>90 \%$ homogeneity as judged by SDS-PAGE. All of the mutants exhibited typical P450 spectra on reduction in the presence of carbon monoxide, with absorbance maxima at $448 \mathrm{~nm}$ and minimal $(<5 \%)$ formation of P420.

\section{Optical Spectroscopy}

UV/Visible spectroscopy was carried out using a Cary 300 Bio UV/visible spectrophotometer equipped with a Peltier temperature control unit and Cary WinUV software. All experiments were conducted in buffer containing $50 \mathrm{mM}$ potassium phosphate, $\mathrm{pH} 8.0$, at $30^{\circ} \mathrm{C}$ unless otherwise stated. Optical titrations 
were conducted by measuring the absorbance changes of the haem Sorêt band on addition of increasing quantities of ligand to a protein solution containing 0.1-0.5 $\mu \mathrm{M}$ P450 in the sample cuvette, and to buffer solution in the reference cuvette. The data were corrected for any dilution of the protein during the course of the experiment, and the change in absorbance difference between $390 \mathrm{~nm}$ and $420 \mathrm{~nm}, \Delta\left(\mathrm{A}_{390}-\mathrm{A}_{420}\right)$, was plotted against substrate concentration, and dissociation constants were estimated using non-linear regression using Microcal Origin software, the protein concentration being measured by CO-difference spectroscopy. ${ }^{54}$

\section{Assay of catalytic activity}

NADPH consumption assays were carried out using reaction mixtures containing 0.1-0.5 $\mu \mathrm{M}$ P450 and varying concentrations of substrates as described. Reactions were initiated by the addition of 200-300 $\mu \mathrm{M}$ NADPH, and the decrease in absorbance at $340 \mathrm{~nm}$ monitored. Initial rates were calculated from the first 20 seconds of the reaction. Apparent $\mathrm{K}_{\mathrm{M}}$ values were estimated by plotting initial rate vs. concentration and fitting with equation 1 ,

$V=V_{0}+\frac{\Delta V_{\max } S}{\left(\mathrm{~K}_{\mathrm{M}}+S\right)}$

a modification of the standard Michaelis-Menten equation which allows for a non-zero reaction rate at zero substrate concentration due to uncoupling reactions. $V_{0}$ is the rate of NADPH consumption in the absence of substrate, and $\Delta V_{\max }$ the maximal increase in rate at saturating substrate concentrations, such that the final maximal observed rate is equal to $\left(V_{\mathrm{o}}+\Delta V_{\max }\right)$. For substrates that showed evidence of cooperativity, a similarly modified version of the Hill equation was used to estimate maximal rates, the substrate concentration giving $50 \%$ of the maximal rate, $S_{0.5}$, and the Hill coefficient, $n_{H}$. In view of the sub-stoichiometric incorporation of haem in some of the mutants, all catalytic rates were normalised to the haem content measured by CO-difference spectroscopy. ${ }^{54}$

\section{Analysis of reaction products}

The products of fatty acid hydroxylation were analysed by NMR using Bruker AMX500 and AMX600 instruments as described previously. ${ }^{55}$ Reactions were carried out in ${ }^{2} \mathrm{H}_{2} \mathrm{O}$ buffer, using $200 \mu$ M NADPH and $200 \mu \mathrm{M}$ laurate such that the concentration of dissolved oxygen (ca. $250 \mu \mathrm{M}$ ) should not be limiting, 
and were initiated by the addition of NADPH. The absorbance at $340 \mathrm{~nm}$ was monitored to ensure the reactions reached completion; NADPH consumption rates in ${ }^{2} \mathrm{H}_{2} \mathrm{O}$ were found to be approximately $90 \%$ of those in water. Reaction mixtures were transferred to NMR tubes and the ${ }^{1} \mathrm{H}-\mathrm{NMR}$ spectra measured directly.

The analysis of the products of indole hydroxylation was carried out separately for the soluble and insoluble products. HPLC analysis of the water-soluble products of the reactions of indole with P450BM3 was carried out as described ${ }^{45}$ using an Agilent 1100 series instrument equipped with diodearray UV/Visible and fluorescence detection, and a Zorbax Reverse Phase SB C-18 $4.6 \times 250 \mathrm{~mm}$ column ( $5 \mu \mathrm{m}$ particle size). All products were identified by demonstration of identical retention volumes to authentic standards, and quantitated by comparison of UV/Visible peak area integrations to known concentrations of authentic standards. The insoluble products were analysed by thin layer chromatography as described ${ }^{46}$ and products identified by comparison to authentic standards of indigo and indirubin. In addition, the insoluble products from a $1 \mathrm{ml}$ reaction containing $1 \mu \mathrm{M}$ P450BM3, $8 \mathrm{mM}$ indole and $300 \mu \mathrm{M}$ NADPH in $50 \mathrm{mM}$ potassium phosphate, $\mathrm{pH} 8$ were redissolved in $600 \mu \mathrm{l}^{2} \mathrm{H}_{6}$-DMSO and the ${ }^{1} \mathrm{H}-\mathrm{NMR}$ spectra were recorded.

\section{Crystallography}

The A82F haem domain was crystallised by the sitting drop method at $4^{\circ} \mathrm{C}$. Sitting drops were prepared by adding $2.5 \mu \mathrm{l}$ of precipitant mixture to $2.5 \mu \mathrm{l}$ of $6 \mathrm{mg} / \mathrm{ml}$ haem domain; crystals were obtained by using a precipitant mixture of $140 \mathrm{mM} \mathrm{MgCl} 2,25 \%$ polyethylene glycol 2000MME and $100 \mathrm{mM}$ MES, $\mathrm{pH}$ 5.0. Crystals were immersed in $10 \%$ polyethylene glycol 200 in mother liquor as a cryoprotectant before being mounted on a rayon loop and flash-cooled in liquid nitrogen. Diffraction data were collected at the European Synchrotron Radiation facility (Grenoble, France) on ID14-EH3 using an ADSC Q4R CCD detector. $180^{\circ}$ data were collected at $100 \mathrm{~K}$ with $1^{\circ}$ oscillations. Data were processed and scaled using MOSFLM $^{56}$ and SCALA $^{57}$. The auto-indexing routines in MOSFLM and examination of reflections along the principal axes established the space group as $\mathrm{P} 22_{1} 2_{1} 2_{1}$ with cell dimensions $a=117.7 \AA \quad b=147.9 \AA \quad c=184.0 \AA$. Molecular replacement calculations were performed with PHASER $1.3,{ }^{58 ;}{ }^{59}$ using a search model based on the substrate-bound wild-type structure (PDB 1JPZ) edited to remove solvent and substrate molecules. Six molecules were found in the asymmetric unit, 
corresponding to a solvent content of $49 \%$. Initial maps clearly showed positive difference density corresponding to the phenolic side chain of residue 82 and to bound substrate. REFMAC5 ${ }^{57 ; 60}$ was used to conduct crystallographic refinement and $\mathrm{COOT}^{61}$ was used for manual rebuilding and density interpretation. The data collection and final refinement parameters are given in Table 4.

\section{Docking calculations}

The protein-ligand docking was performed using GOLD3.1.1 ${ }^{62}$ without any constraints. The template protein PDB files used were 1JPZ for the wild-type enzyme and $2 \mathrm{UWH}$ for the A82F mutant. The ligands were then docked into a sphere of radius $15 \AA$ around the iron centre of haem.

\section{Accession numbers}

The refined coordinates and structure factors have been deposited with the protein databank (PDB 2UWH).

\section{ACKNOWLEDGEMENTS}

This work was supported by the BBSRC (grant E20186) and by an ORSAS grant to W.-C. H. We are grateful to Professors A.W. Munro and Paul M. Cullis for valuable discussions at the start of this project, to Professor Luet Wong for the gift of the pGLWBM3 plasmid, to Dr. Fred Muskett for help with the NMR experiments and to Professor Peter Farmer and Dr. Don Jones for the LC-MS analyses. 
TABLE 1

Binding and kinetic parameters for fatty acid binding to wild-type and mutant P450BM3

\begin{tabular}{|l|c|c|c|c|c|c|}
\hline \multirow{2}{*}{ Enzyme } & \multicolumn{5}{|c|}{ Laurate } & \multicolumn{2}{c|}{ Palmitate } \\
\hline & $K_{\mathbf{d}}(\mu \mathbf{M})^{\mathbf{1}}$ & $\begin{array}{c}\% \text { high } \\
\text { spin }\end{array}$ & $K_{\mathbf{M}}(\mu \mathbf{M})^{2}$ & $\mathbf{k}_{\text {cat }}\left(\mathbf{s}^{-1}\right)^{2}$ & $K_{\mathbf{d}}(\mu \mathbf{M})^{\mathbf{1}}$ & $\begin{array}{c}\% \text { high } \\
\text { spin }\end{array}$ \\
\hline Wild-type & $270( \pm 14)$ & 53 & $265( \pm 19)$ & $28( \pm 1)$ & $0.14( \pm 0.01)$ & 87 \\
\hline A82I & $240( \pm 17)$ & 50 & $320( \pm 16)$ & $45( \pm 1)$ & $0.27( \pm 0.01)$ & 85 \\
\hline A82F & $0.34( \pm 0.03)$ & 92 & $<20$ & $26( \pm 1)$ & $<0.1$ & 100 \\
\hline A82W & $0.43( \pm 0.04)$ & 93 & $<20$ & $43( \pm 1)$ & $<0.1$ & 100 \\
\hline
\end{tabular}

${ }^{\mathbf{1}}$ From optical titration experiments; see Materials and Methods.

${ }^{2}$ From measurements of NADPH consumption; see Materials and Methods. 


\section{TABLE 2}

Indole binding and turnover by wild-type and mutant cytochrome P450BM3.

\begin{tabular}{|c|c|c|c|c|c|}
\hline \multirow{2}{*}{ Enzyme } & \multicolumn{2}{|c|}{ Indole binding } & \multicolumn{2}{c|}{ Indole turnover $^{2}$} \\
\cline { 2 - 6 } & $\mathbf{K}_{\mathbf{d}} / \mathbf{K}_{\mathrm{d} 1}$ & $\mathbf{K}_{\mathrm{d} 2}$ & $K_{\mathrm{M}}(\mathrm{mM})$ & $n_{H}$ & $\Delta \mathbf{V}_{\text {max }}\left(\mathbf{s}^{-1}\right)$ \\
\hline Wild-type & $11( \pm 5) \mathrm{mM}$ & - & $16( \pm 5)$ & $1.7( \pm 0.3)$ & $3.7 \pm 0.9$ \\
\hline A82I & $13( \pm 7) \mathrm{mM}$ & - & $14( \pm 4)$ & $1.8( \pm 0.4)$ & $5.5 \pm 1.4$ \\
\hline A82F & $0.1( \pm 0.04) \mu \mathrm{M}$ & $317( \pm 91) \mu \mathrm{M}$ & $3.4( \pm 0.9)$ & $1.2( \pm 0.2)$ & $20.8 \pm 2.6$ \\
\hline A82W & $0.08( \pm 0.05) \mu \mathrm{M}$ & $168( \pm 62) \mu \mathrm{M}$ & $0.2( \pm 0.1)$ & $0.6( \pm 0.1)$ & $25.9 \pm 2.8$ \\
\hline
\end{tabular}

${ }^{1}$ From optical titration experiments; see Materials and Methods.

${ }^{2}$ From measurements of NADPH consumption, fit by the modified Hill equation as described in the ;

Materials and Methods section. Note that $\Delta \mathrm{V}_{\max }$ is the increase in the rate of NADPH consumption on addition of saturating indole concentrations, not the rate of product formation; for the latter, see Table 3 


\section{TABLE 3}

Product formation and NADPH consumption during indole hydroxylation by wild-type and mutant cytochrome P450BM3

\begin{tabular}{|c|c|c|c|c|c|}
\hline Enzyme & $\begin{array}{c}\text { Indigo } \\
\text { formed }^{1} \\
(\mu \mathrm{M})\end{array}$ & $\begin{array}{l}\text { Oxindole } \\
\text { formed }^{1} \\
(\mu \mathrm{M})\end{array}$ & $\begin{array}{l}\text { Coupling of product } \\
\text { formation to } \\
\text { NADPH } \\
\text { consumption }(\%)^{2}\end{array}$ & $\begin{array}{c}\text { Rate of } \\
\text { NADPH } \\
\text { consumption } \\
\left(\mathrm{s}^{-1}\right)\end{array}$ & $\begin{array}{c}\text { Rate of } \\
\text { product } \\
\text { formation }^{3} \\
{\left(\mathrm{~s}^{-1}\right)}\end{array}$ \\
\hline Wild-type & $2.5( \pm 0.9)$ & $7( \pm 0.6)$ & $4( \pm 1.4)$ & 1.1 & 0.03 \\
\hline A82I & $11( \pm 2.8)$ & $12( \pm 0.4)$ & $11( \pm 2.6)$ & 1.5 & 0.19 \\
\hline A82F & $41( \pm 8.8)$ & $34( \pm 2.0)$ & $39( \pm 9)$ & 16 & 6.0 \\
\hline A82W & $22( \pm 2.3)$ & $48( \pm 1.1)$ & $31( \pm 8)$ & 23 & 5.7 \\
\hline
\end{tabular}

${ }^{1}$ Reaction mixtures contained $1 \mu \mathrm{M}$ enzyme, $8 \mathrm{mM}$ indole and $300 \mu \mathrm{M}$ NADPH in $50 \mathrm{mM}$ potassium phosphate, $\mathrm{pH} 8.0$; products were measured on completion of the reaction. Indigo formation was estimated from UV/Vis absorbance of insoluble products redissolved in DMSO and oxindole formation by peak area integration of the HPLC chromatogram of the soluble products; see Materials and Methods section and Supplementary Information.

${ }^{2}$ Since one molecule of indigo is formed from two molecules of the precursor product indoxyl (Scheme 1), the percentage coupling is defined as $100 \times(([$ oxindole formed $]+2 \times$ [indigo formed $]) /$ [NADPH consumed])

${ }^{3}$ Calculated from the NADPH consumption rate and the percentage coupling. 


\section{TABLE 4}

\section{Crystallographic statistics}

Statistics of Data Collection and Refinement (Values in parentheses refer to the outer bin.)

\begin{tabular}{|c|c|}
\hline Space group & $\mathrm{P} 22_{1} 2_{1}$ \\
\hline Cell dimensions & $\begin{array}{ll}\mathrm{a}=116.8 \AA & \alpha=90^{\circ} \\
\mathrm{b}=147.0 \AA & \beta=90^{\circ} \\
\mathrm{c}=183.4 \AA & \gamma=90^{\circ}\end{array}$ \\
\hline Data range $(\AA)$ & $2.80-73.52(2.80-2.95)$ \\
\hline Number of measurements & $440872(57179)$ \\
\hline Number of independent reflections & $76791(10567)$ \\
\hline Mean $((\mathrm{I}) / \mathrm{sd}(\mathrm{I}))$ & $10.6(2.5)$ \\
\hline $\mathrm{R}_{\text {merge }}(\%)$ & $0.185(0.638)$ \\
\hline Data completeness $(\%)$ & $98.0(93.7)$ \\
\hline Number of non-hydrogen atoms & 23027 \\
\hline $\begin{array}{l}\text { Number of water molecules in the } \\
\text { asymmetric unit }\end{array}$ & 521 \\
\hline Number of reflections used in refinement & 72833 \\
\hline $\mathrm{R}_{\mathrm{work}}$ & 0.22 \\
\hline $\mathrm{R}_{\text {free }}$ & 0.30 \\
\hline ESU based on free R value $(\AA)$ & 0.482 \\
\hline RMSD in bond length $(\AA)$ & 0.008 \\
\hline RMSD in bond angle $\left(^{\circ}\right)$ & 1.383 \\
\hline Missing residues in chains A, B, C, D, E, F & $459-472$ \\
\hline $\begin{array}{l}\text { Analysis of Ramachandran plot (using } \\
\text { PROCHECK }^{63} \text { ) }\end{array}$ & $\begin{array}{l}\text { Most-favoured regions } \\
86.5 \% \\
\text { Additional allowed } \\
\text { regions } 11.6 \% \\
\text { Generously allowed } \\
\text { regions } 1 \%\end{array}$ \\
\hline Mean B factor & $22.8 \AA^{2}$ \\
\hline
\end{tabular}




\section{REFERENCES}

1. Makris, T. M., Denisov, I., Schlichting, I., and Sligar, S.G. (2005). Activation of molecular oxygen by cytochrome P450. In Cytochrome P450: Structure, Mechanism and Biochemistry 3rd. edit. (Ortiz de Montellano, P. R., ed.), pp. 149-182. Kluwer Academic / Plenum Publishers, New York.

2. Ortiz de Montellano, P. R., and De Voss, J. J. (2005). Substrate oxidation by cytochrome P450 enzymes. In Cytochrome P450: Structure, Mechanism and Biochemistry 3rd edit. (Ortiz de Montellano, P. R., ed.), pp. 183-246. Kluwer Academic / Plenum Publishers, New York.

3. Guengerich, F. P. (2005). Human cytochrome P450 enzymes. In Cytochrome P450: Structure, Mechanism and Biochemistry Third edit. (Ortiz de Montellano, P. R., ed.), pp. 377-530. Kluwer Academic/Plenum, New York.

4. Kelly, S. L., Kelly, D.E., Jackson, C.L., Warrilow, A.G.S., and Lamb, D.C. (2005). The diversity and importance of microbial cytochromes P450. In Cytochrome P450: Structure, mechanism and biochemistry (Ortiz de Montellano, P. R., ed.), pp. 585-618. Kluwer Academic / Plenum Publishing, New York.

5. Nielsen, K. A., and Moller, B.L. (2005). Cytochrome P450s in plants. In Cytochrome P450: Structure, mechanism and biochemistry (Ortiz de Montellano, P. R., ed.), pp. 553-584. Kluwer Academic / Plenum Publishers, New York.

6. Warren, J. T., Petryk, A., Marques, G., Jarcho, M., Parvy, J. P., Dauphin-Villemant, C., O'Connor, M. B. \& Gilbert, L. I. (2002). Molecular and biochemical characterization of two P450 enzymes in the ecdysteroidogenic pathway of Drosophila melanogaster. Proc. Nat.Acad. Sci.USA 99, 11043-11048.

7. Munro, A. W., Leys, D. G., McLean, K. J., Marshall, K. R., Ost, T. W. B., Daff, S., Miles, C. S., Chapman, S. K., Lysek, D. A., Moser, C. C., Page, C. C. \& Dutton, P. L. (2002). P450 BM3: the very model of a modern flavocytochrome. Trends Biochem. Sci. 27, 250-257.

8. Poulos, T. L. (2005). Intermediates in P450 catalysis. Phil.l Trans. Roy. Soc. Lond A 363, 793806. 
9. Pylypenko, O. \& Schlichting, I. (2004). Structural aspects of ligand binding to and electron transfer in bacterial and fungal P450s. Ann. Rev. Biochem. 73, 991-1018.

10. Narhi, L. O. \& Fulco, A. J. (1986). Characterization of a catalytically self-sufficient 119,000dalton cytochrome P-450 monooxygenase induced by barbiturates in Bacillus megaterium. J. Biol. Chem. 261, 7160-7169.

11. Fulco, A. J. (1991). P450BM-3 and other inducible bacterial P450 cytochromes: Biochemistry and regulation. Ann. Rev. Pharmacol. Toxicol. 31, 177-203.

12. Narhi, L. O. \& Fulco, A. J. (1987). Identification and characterization of two functional domains in cytochrome P-450BM-3, a catalytically self-sufficient monooxygenase induced by barbiturates in Bacillus megaterium. J. Biol. Chem. 262, 6683-6690.

13. Ravichandran, K. G., Boddupalli, S. S., Hasemann, C. A., Peterson, J. A. \& Deisenhofer, J. (1993). Crystal structure of hemoprotein domain of P450BM-3, a prototype for microsomal P450's. Science 261, 731-736.

14. Li, H. Y. \& Poulos, T. L. (1997). The structure of the cytochrome P450BM-3 haem domain complexed with the fatty acid substrate, palmitoleic acid. Nature Struct. Biol. 4, 140-146.

15. Haines, D. C., Tomchick, D. R., Machius, M. \& Peterson, J. A. (2001). Pivotal role of water in the mechanism of P450BM-3. Biochemistry 40, 13456-13465.

16. Joyce, M. G., Girvan, H. M., Munro, A. W. \& Leys, D. (2004). A single mutation in cytochrome P450 BM3 induces the conformational rearrangement seen upon substrate binding in the wildtype enzyme. J. Biol. Chem. 279, 23287-23293.

17. Ekroos, M., and Sjogren, T. (2006). Structural basis for ligand promiscuity in cytochrome P450 3A4. Proc. Nat. Acad. Sci. USA 103, 13682-13687.

18. Williams, P. A., Cosme, J., Vinkovic, D. M., Ward, A., Angove, H. C., Day, P. J., Vonrhein, C., Tickle, I. J. \& Jhoti, H. (2004). Crystal structures of human cytochrome P450 3A4 bound to metyrapone and progesterone. Science 305, 683-686.

19. Williams, P. A., Cosme, J., Ward, A., Angova, H. C., Vinkovic, D. M. \& Jhoti, H. (2003). Crystal structure of human cytochrome P4502C9 with bound warfarin. Nature 424, 464-468. 
20. Li, H. Y., Narasimhulu, S., Havran, L. M., Winkler, J. D. \& Poulos, T. L. (1995). Crystal structure of cytochrome P450cam complexed with its catalytic product, 5-exo-hydroxycamphor. J. Amer. Chem. Soc. 117, 6297-6299.

21. Raag, R. \& Poulos, T. L. (1989). Crystal structure of the carbon monoxide-substrate-cytochrome P-450 CAM ternary complex. Biochemistry 28, 7586-7592.

22. Cupp-Vickery, J. R. \& Poulos, T. L. (1995). Structure of cytochrome P450eryf involved in erythromycin biosynthesis. Nature Struct. Biol. 2, 144-153.

23. Nagano, S., Li, H. Y., Shimizu, H., Nishida, C., Ogura, H., de Montellano, P. R. O. \& Poulos, T. L. (2003). Crystal structures of epothilone D-bound, epothilone B-bound, and substrate-free forms of cytochrome P450epoK. J. Biol. Chem. 278, 44886-44893.

24. Modi, S., Primrose, W. U., Boyle, J. M. B., Gibson, C. F., Lian, L. Y. \& Roberts, G. C. K. (1995). NMR studies of substrate binding to cytochrome P450 BM3: comparisons to cytochrome P450 cam. Biochemistry 34, 8982-8988.

25. Jovanovic, T. \& McDermott, A. E. (2005). Observation of ligand binding to cytochrome P450 BM-3 by means of solid-state NMR spectroscopy. J. Amer. Chem. Soc. 127, 13816-13821.

26. Jovanovic, T., Farid, R., Friesner, R. A. \& McDermott, A. E. (2005). Thermal equilibrium of high- and low-spin forms of cytochrome P450 BM-3: Repositioning of the substrate? J. Amer. Chem. Soc. 127, 13548-13552.

27. Ravindranathan, K. P., Gallicchio, E., Friesner, R. A., McDermott, A. E. \& Levy, R. M. (2006). Conformational equilibrium of cytochrome P450 BM-3 complexed with N-palmitoylglycine: A replica exchange molecular dynamics study. J. Amer. Chem. Soc. 128, 5786-5791.

28. Carmichael, A. B. \& Wong, L. L. (2001). Protein engineering of Bacillus megaterium CYP102 The oxidation of polycyclic aromatic hydrocarbons. Eur. J. Biochem. 268, 3117-3125.

29. Appel, D., Lutz-Wahl, S., Fischer, P., Schwaneberg, U. \& Schmid, R. D. (2001). A P450 BM-3 mutant hydroxylates alkanes, cycloalkanes, arenes and heteroarenes. J. Biotechnol. 88, 167-171.

30. Cirino, P. C. \& Arnold, F. H. (2002). Regioselectivity and activity of cytochrome P450 BM-3 and mutant F87A in reactions driven by hydrogen peroxide. Adv. Synth. Catal. 344, 932-937. 
31. Cowart, L. A., Falck, J. R. \& Capdevila, J. H. (2001). Structural determinants of active site binding affinity and metabolism by cytochrome P450 BM-3. Arch. Biochem. Biophys. 387, 117124.

32. Graham-Lorence, S., Truan, G., Peterson, J. A., Falck, J. R., Wei, S. Z., Helvig, C. \& Capdevila, J. H. (1997). An active site substitution, F87V, converts cytochrome P450 BM-3 into a regioand stereoselective (14S,15R)-arachidonic acid epoxygenase. J. Biol. Chem. 272, 1127-1135.

33. Li, Q. S., Ogawa, J., Schmid, R. D. \& Shimizu, S. (2001). Residue size at position 87 of cytochrome P450BM-3 determines its stereoselectivity in propylbenzene and 3-chlorostyrene oxidation. FEBS Letts. 508, 249-252.

34. Sulistyaningdyah, W. T., Ogawa, J., Li, Q. S., Maeda, C., Yano, Y., Schmid, R. D. \& Shimizu, S. (2005). Hydroxylation activity of P450BM-3 mutant F87V towards aromatic compounds and its application to the synthesis of hydroquinone derivatives from phenolic compounds. Appl. Microbiol. Biotechnol. 67, 556-562.

35. Munro, A. W., Daff, S., Coggins, J. R., Lindsay, J. G. \& Chapman, S. K. (1996). Probing electron transfer in flavocytochrome P-450 BM3 and its component domains. Eur. J. Biochem.239, 403-409.

36. Neeli, R., Girvan, H. M., Lawrence, A., Warren, M. J., Leys, D., Scrutton, N. S. \& Munro, A. W. (2005). The dimeric form of flavocytochrome P450 BM3 is catalytically functional as a fatty acid hydroxylase. FEBS Letts. 579, 5582-5588.

37. Magnuson, K., Jackowski, S., Rock, C. O. \& Cronan, J. E., Jr. (1993). Regulation of fatty acid biosynthesis in Escherichia coli. Microbiol. Rev. 57, 522-542.

38. Yeom, H., Sligar, S. G., Li, H. Y., Poulos, T. L. \& Fulco, A. J. (1995). The role of Thr268 in oxygen activation of cytochrome P450BM-3. Biochemistry 34, 14733-14740.

39. Raag, R. \& Poulos, T. L. (1991). Crystal structures of cytochrome P-450 $0_{\text {CAM }}$ complexed with camphane, thiocamphor, and adamantane: factors controlling P-450 substrate hydroxylation. Biochemistry 30, 2674-2684.

40. Modi, S., Sutcliffe, M. J., Primrose, W. U., Lian, L. Y. \& Roberts, G. C. K. (1996). The catalytic mechanism of cytochrome P450 BM3 involves a $6 \AA$ movement of the bound substrate on reduction. Nature Struct. Biol. 3, 414-417. 
41. Helms, V., Deprez, E., Gill, E., Barret, C., Hoa, G. H. B. \& Wade, R. C. (1996). Improved binding of cytochrome P450cam substrate analogues designed to fill extra space in the substrate binding pocket. Biochemistry 35, 1485-1499.

42. Rock, D. A., Boitano, A. E., Wahlstrom, J. L., Rock, D. A. \& Jones, J. P. (2002). Use of kinetic isotope effects to delineate the role of phenylalanine 87 in P450(BM-3). Bioorg. Chem. 30, 107118.

43. Noble, M. A., Miles, C. S., Chapman, S. K., Lysek, D. A., Mackay, A. C., Reid, G. A., Hanzlik, R. P. \& Munro, A. W. (1999). Roles of key active-site residues in flavocytochrome P450 BM3. Biochem. J. 339, 371-379.

44. Gillam, E. M. J., Aguinaldo, A. M. A., Notley, L. M., Kim, D., Mundkowski, R. G., Volkov, A. A., Arnold, F. H., Soucek, P., DeVoss, J. J. \& Guengerich, F. P. (1999). Formation of indigo by recombinant mammalian cytochrome P450. Biochem. Biophys. Res. Commun. 265, 469-472.

45. Gillam, E. M. J., Notley, L. M., Cai, H. L., De Voss, J. J. \& Guengerich, F. P. (2000). Oxidation of indole by cytochrome P450 enzymes. Biochemistry 39, 13817-13824.

46. Li, Q. S., Schwaneberg, U., Fischer, P. \& Schmid, R. D. (2000). Directed evolution of the fattyacid hydroxylase P450 BM-3 into an indole-hydroxylating catalyst. Chem. Eur. J. 6, 1531-1536.

47. Nakamura, K., Martin, M. V. \& Guengerich, F. P. (2001). Random mutagenesis of human cytochrome P450 2A6 and screening with indole oxidation products. Arch. Biochem. Biophys. $395,25-31$.

48. Li, Q. S., Ogawa, J., Schmid, R. D. \& Shimizu, S. (2005). Indole hydroxylation by bacterial cytochrome P450 BM-3 and modulation of activity by cumene hydroperoxide. Biosci. Biotechnol. Biochem. 69, 293-300.

49. van Vugt-Lussenburg, B. M. A., Damsten, M. C., Maasdijk, D. M., Vermeulen, N. P. E. \& Commandeur, J. N. M. (2006). Heterotropic and homotropic cooperativity by a drugmetabolising mutant of cytochrome P450 BM3. Biochem. Biophys. Res. Commun. 346, 810-818.

50. Rock, D. A., Perkins, B. N. S., Wahlstrom, J. \& Jones, J. P. (2003). A method for determining two substrates binding in the same active site of cytochrome P450BM3: an explanation of high energy omega product formation. Arch. Biochem. Biophys. 416, 9-16.

51. Bernhardt, R. (2006). Cytochromes P450 as versatile biocatalysts. J. Biotechnol. 124, 128-145. 
52. Urlacher, V. B., Makhsumkhanov, A. \& Schmid, R. D. (2006). Biotransformation of beta-ionone by engineered cytochrome P450 BM-3. Appl. Microbiol. Biotechnol. 70, 53-59.

53. Li, Q. S., Ogawa, J., Schmid, R. D. \& Shimizu, S. (2001). Engineering cytochrome P450BM-3 for oxidation of polycyclic aromatic hydrocarbons. Appl. Environ. Microbiol.67, 5735-5739.

54. Omura, T. \& Sato, R. (1964). The carbon monoxide-binding pigment of liver microsomes II. Solubilization, purification, and properties. J. Biol. Chem. 239, 2379-2385.

55. Oliver, C. F., Modi, S., Sutcliffe, M. J., Primrose, W. U., Lian, L. Y. \& Roberts, G. C. K. (1997). A single mutation in cytochrome P450 BM3 changes substrate orientation in a catalytic intermediate and the regiospecificity of hydroxylation. Biochemistry 36, 1567-1572.

56. Leslie, A. G. W. (1992). Recent changes to the MOSFLM package for processing film and image plate data. In CCP4 \& ESF-EAMCB Newslett. Protein Crystallog.

57. Collaborative_Computational_Project. (1994). The CCP4 suite: programs for protein crystallography. Acta Cryst. D50, 760-763.

58. Storoni, L. C., McCoy, A.J., and Read, R.J. (2004). Likelihood-enhanced fast rotation functions. Acta Cryst. D60, 432-438.

59. McCoy, A. J., Grosse-Kunstleve, R.W., Storoni, L.C., and Read, R.J. (2005). Likelihoodenhanced fast translation functions. Acta Cryst. D61, 458-464.

60. Potterton, E., Briggs, P., Turkenburg, M., and Dodson, E. (2003). A graphical user interface to the CCP4 program suite. Acta Cryst. D59, 1131-1137.

61. Emsley, P., and Cowtan, K. (2004). COOT: model-building tools for molecular graphics. Acta Crystallographica D60, 2126-2132.

62. Verdonk, M. L., Cole, J.C., Hartshorn, M.J., Murray, C.W., Taylor, R.D. (2003). Improved protein-ligand docking using GOLD. Proteins: Struct. Funct. Genet. 52, 609-623.

63. Laskowski, R. A., MacArthur, M.W., Moss, D.S., and Thornton, J.M. . (1993). PROCHECK: a program to check the stereochemical quality of protein structures. J. Appl. Cryst. 26, 283-291. 


\section{FIGURE LEGENDS}

\section{Figure 1}

The active site of the N-palmitoyl-glycine complex of the wild-type enzyme (A; PDB 1JPZ) and of models of the mutants with isoleucine (B), phenylalanine (C) or tryptophan (D) in place of alanine at position 82.

The side chain at position 82 is shown in yellow, and the van der Waals surface of the substrate-binding pocket in magenta. A space-filling representation of the substrate N-palmitoylglycine is superimposed in blue, occupying the position observed in the crystal structure. The modelled structures in B-D show the larger side-chains at position 82 filling the hydrophobic pocket discussed in the text and overlapping with the bound fatty acid

\section{Figure 2}

\section{Fatty acid binding to wild-type and mutant enzyme}

Top: Changes in UV/Visible absorption spectra on fatty acid binding for (A) Wild-type enzyme, (B) A82I, (C) A82F, (D) A82W mutants. In each case spectra are shown for $10 \mu \mathrm{M}$ enzyme without substrate (blue), and in the presence of $2 \mathrm{mM}$ laurate (orange) or $10 \mu \mathrm{M}$ palmitate (red).

Bottom: Concentration dependence of the optical absorption change on laurate binding to (E) $2.5 \mu \mathrm{M}$ wild-type enzyme and (F) $0.25 \mu \mathrm{M}$ A82F enzyme. Note the difference in enzyme concentration and in substrate concentration scale between $(\mathrm{E})$ and $(\mathrm{F})$.

\section{Figure 3}

\section{${ }^{1} \mathrm{H}$ NMR spectra of the products of hydroxylation of laurate by wild-type and mutant P450BM3}

The methyl region of the spectra is shown for (A-D) reaction mixtures containing (A) A82W, (B) A82F, (C) A82I, and (D) wild type enzyme, and (E) a laurate standard.

The methyl resonances of laurate and its hydroxylation products are identified as (1) 11-hydroxylaurate $(\delta=1.071, \mathrm{M}=2, \mathrm{~J}=6.24 \mathrm{~Hz}),(2)$ 10-hydroxylaurate $(\delta=0.808, \mathrm{M}=3, \mathrm{~J}=7.17 \mathrm{~Hz}),(3)$ 9-hydroxylaurate $(\delta=0.803, \mathrm{M}=3, \mathrm{~J}=7.53 \mathrm{~Hz})$, and (4) laurate $(\delta=0.775, \mathrm{M}=3, \mathrm{~J}=6.68 \mathrm{~Hz})$ 
The triplet partially overlapping the 11-hydroxylaurate methyl resonance $(\delta=1.096, \mathrm{M}=3, \mathrm{~J}=7.15 \mathrm{~Hz})$ is from an impurity in the NADPH, and is also present in control reactions containing no substrate.

\section{Figure 4}

\section{Structure of the palmitate complex of the haem domain of the A82F mutant of P450BM3}

A. Overall structure of the palmitate complex of the A82F mutant (cyan) compared to the Npalmitoylglycine complex of the wild-type enzyme (PDB 1JPZ; green). The haem group is shown in red, the palmitate bound to $\mathrm{A} 82 \mathrm{~F}$ in brown and the N-palmitoylglycine bound to wild-type in purple; the mutated Phe82 residue is highlighted in yellow. The F and $\mathrm{G}$ helices are indicated.

B. Stereo view of the active site in the crystal structure of the A82F mutant. The side chain of the mutated Phe82 residue is highlighted in magenta. The bound palmitate is in dark blue, the haem in red and Tyr51, Phe87 and Phe81 are in yellow. The blue mesh is the electron density map calculated using the omit procedure (2 cycles) in SFCHECK ${ }^{57}$. The omit maps for all six chains in the asymmetric unit are shown in the Supplementary material.

C. Comparison of the substrate binding site in the substrate-free and substrate bound wild-type enzyme with that in the substrate-bound A82F mutant. Relevant substrate contact residues are shown. The A82F mutant with palmitate bound ( $2 \mathrm{UWH})$ is in pink, the wild-type with N-palmitoylglycine bound (1JPZ) is in cyan and the wild-type without substrate $(2 \mathrm{BMH})$ is in green.

\section{Figure 5}

Temperature dependence of the optical absorption spectra of the palmitate complexes of wild-type and mutant P450BM3

Spectra are shown for (A) wild-type enzyme, and (B) A82I, (C)A82F, and (D) A82W mutants. In each case, spectra shown were recorded at $30^{\circ} \mathrm{C}$ (red), $20^{\circ} \mathrm{C}$ (orange), $10^{\circ} \mathrm{C}$ (light blue), and $4^{\circ} \mathrm{C}$ (dark blue). 


\section{Figure 6}

\section{Displacement of Ile263 on substrate binding.}

A, Wild-type N-palmitoylglycine-bound (PDB 1JPZ); B, Wild-type substrate-free (PDB 2BMH); C, A82F palmitate bound; D, A82F modelled into the wild-type substrate-free structure, demonstrating the clash between F82 and L263 in this conformation

Residue I263 is highlighted in dark blue and residue 82 in yellow. The other residues shown are F81 and F87 above and below (A/F)82, and residues on the F helix, F173, M177 and L181.

\section{Figure 7}

\section{Indole binding to wild-type and mutant enzyme}

Changes in UV/Visible absorption spectra of wild-type enzyme (top) and A82F enzyme (bottom) on indole binding. Spectra are shown for enzyme without substrate (blue), and in the presence of $8 \mathrm{mM}$ indole (orange) or $18 \mathrm{mM}$ indole (red).

\section{Figure 8}

\section{Docking of indole into the active site of P450 BM3}

Indole is shown as a space-filling model, docked into the active site of (A) the wild-type enzyme (1JPZ) and (B) the A82F mutant (2UWH). Sidechains of F81 (yellow), F82/A82 (pink) and F87 (green) are shown. Docking was performed by using GOLD3.1.1 ${ }^{62}$. 
Figure 1
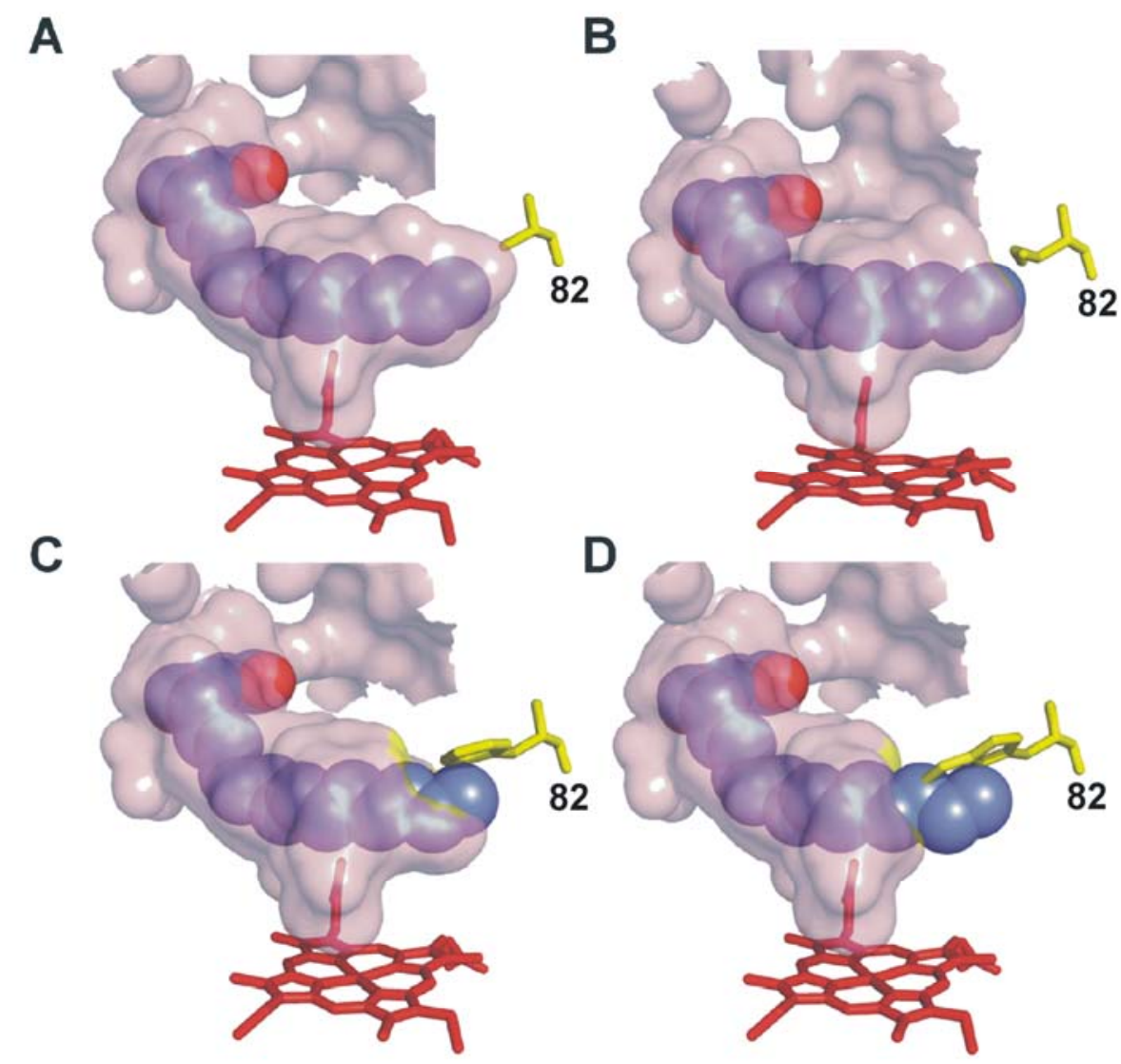

Figure 2
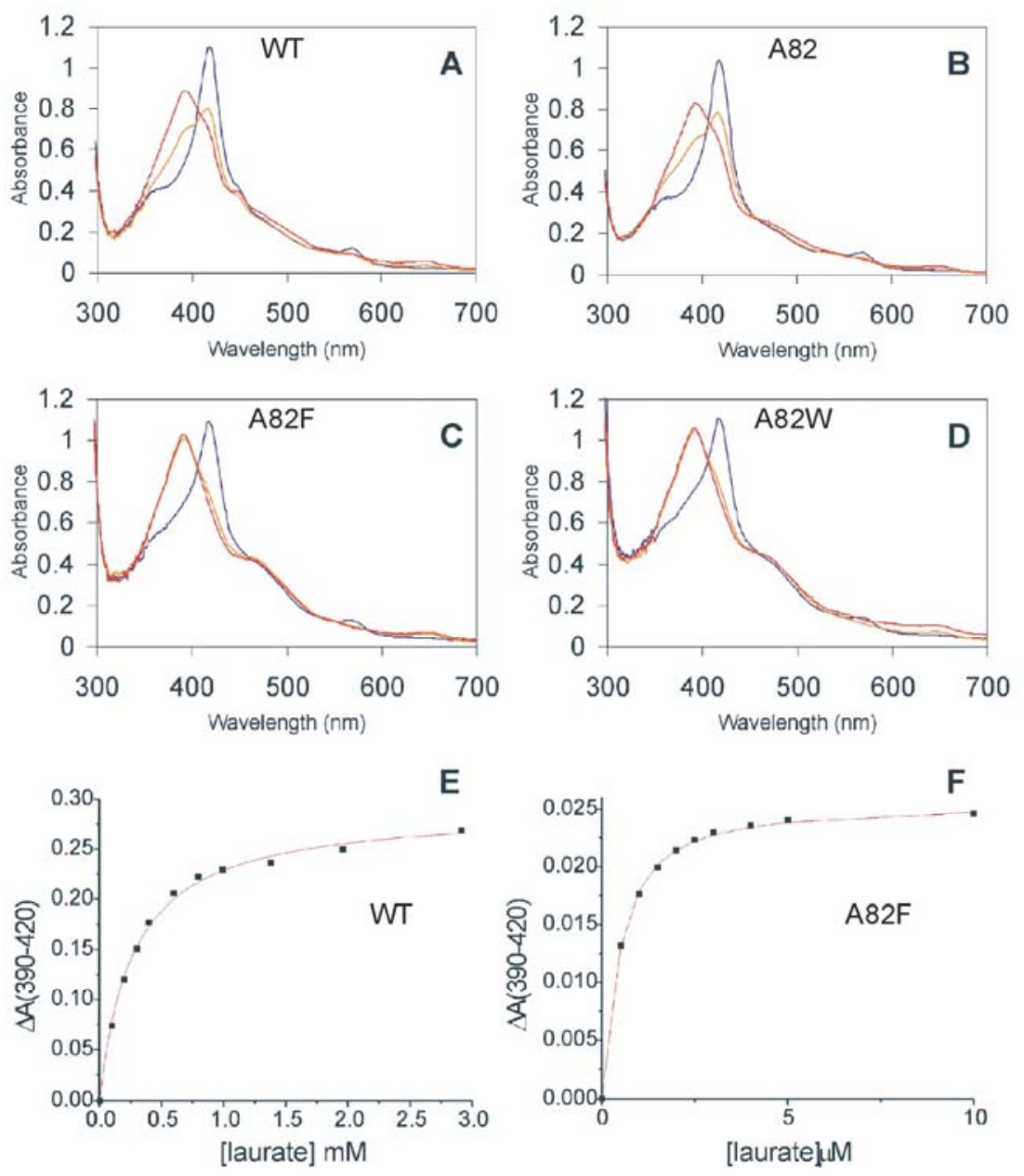
Figure 3

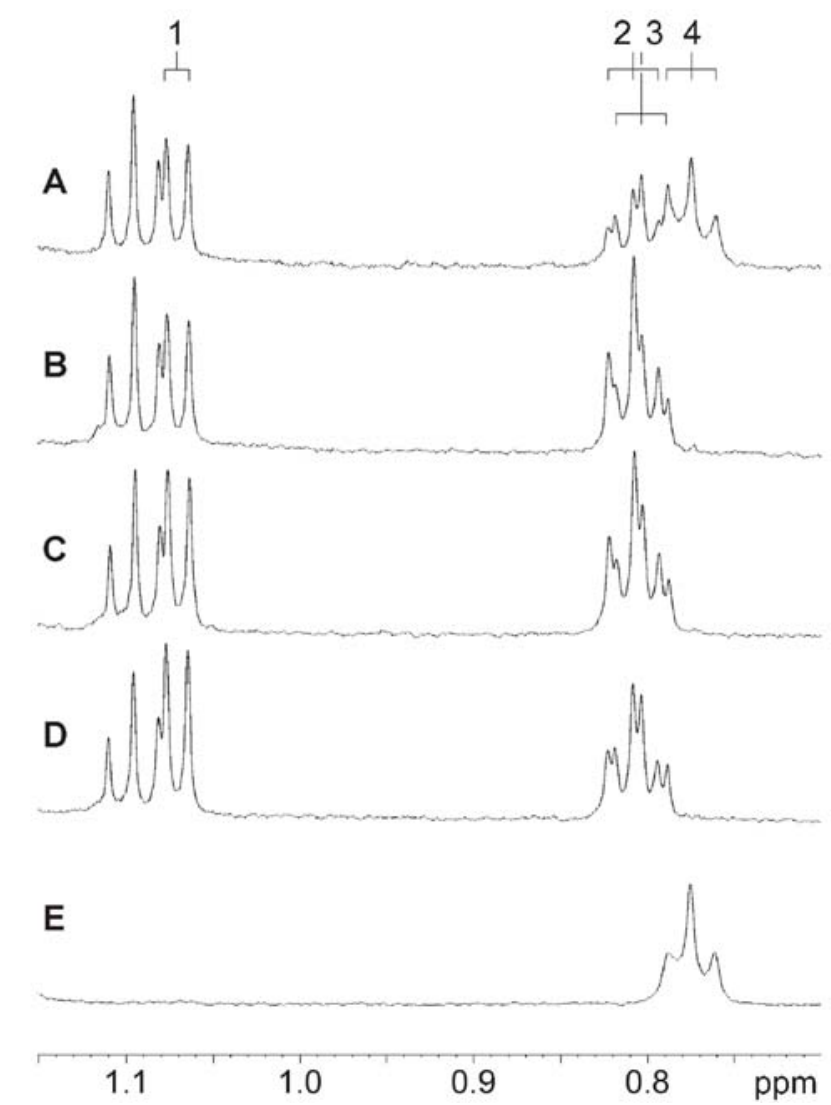

Figure 4A

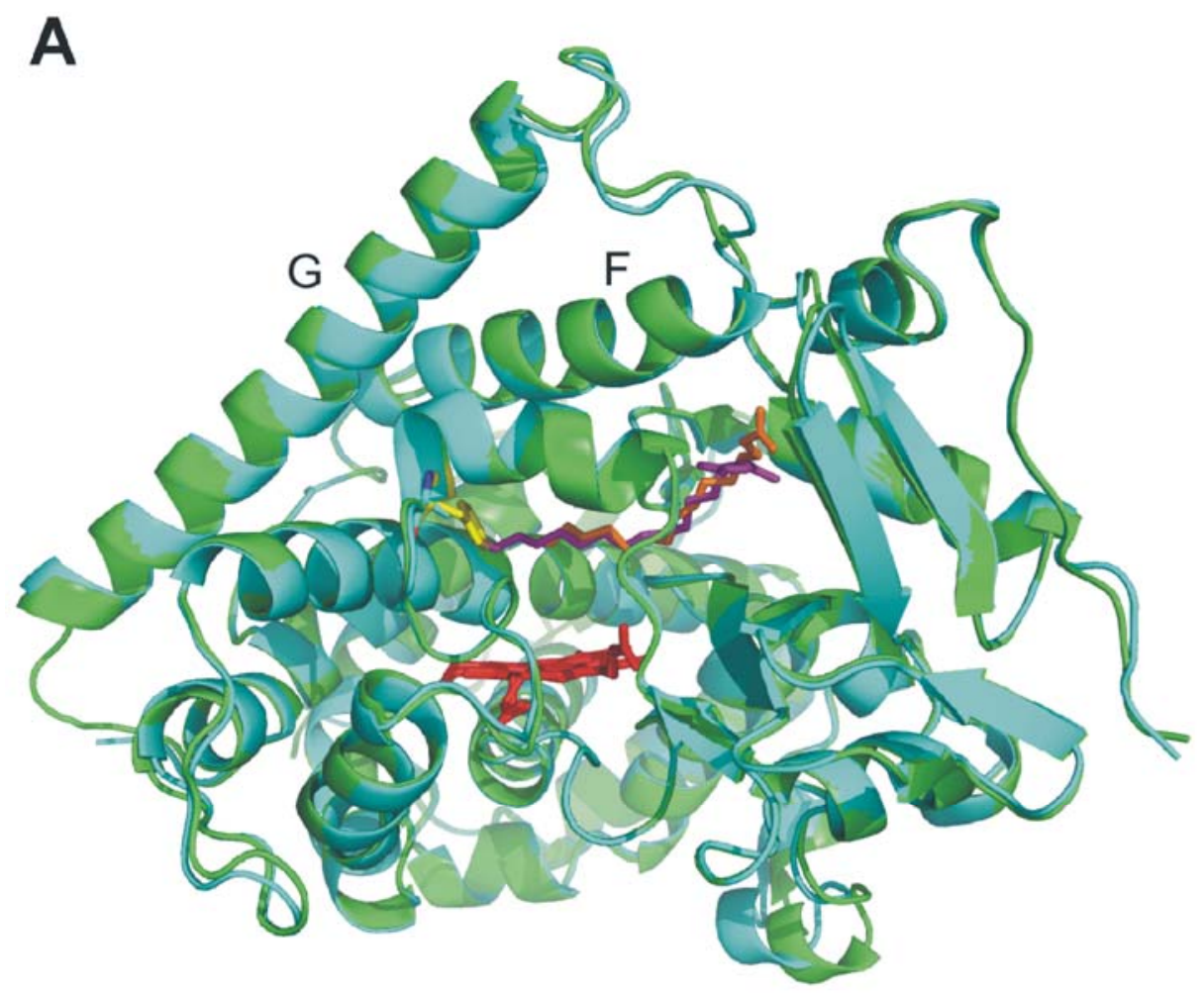


Figure 4B

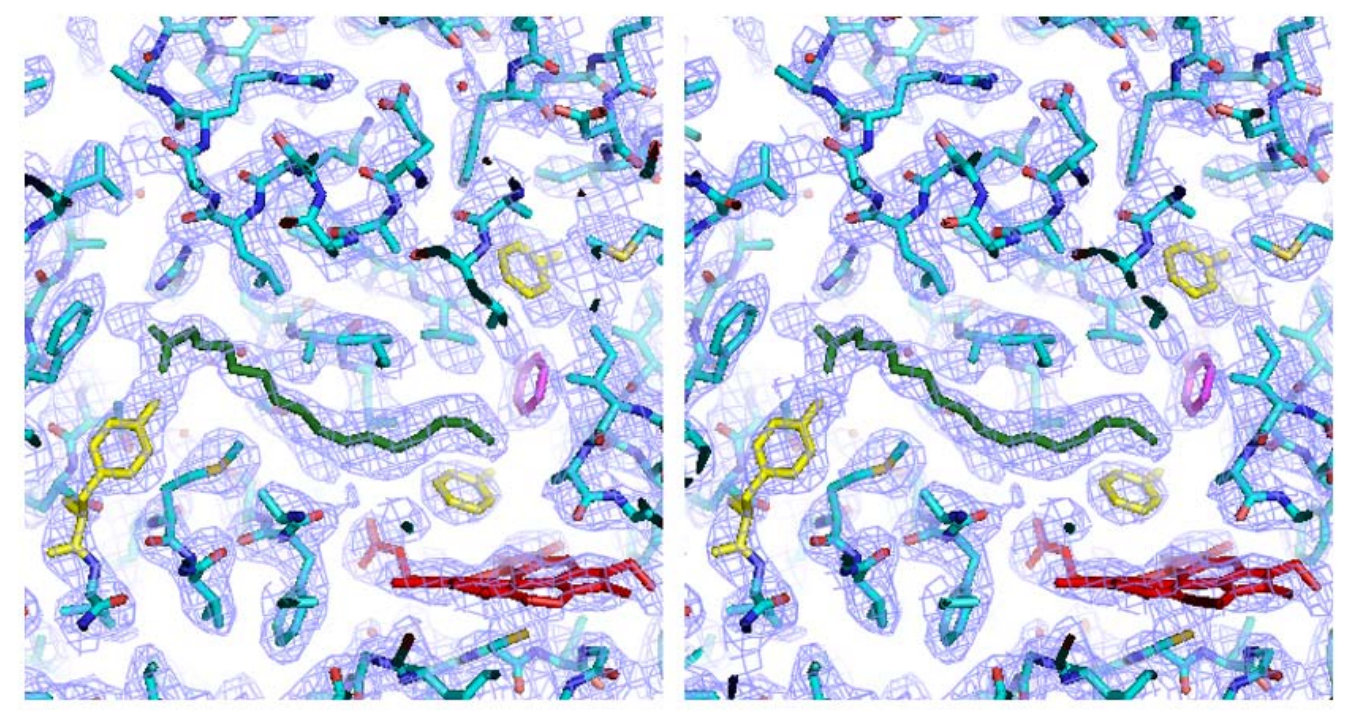

Figure 4C

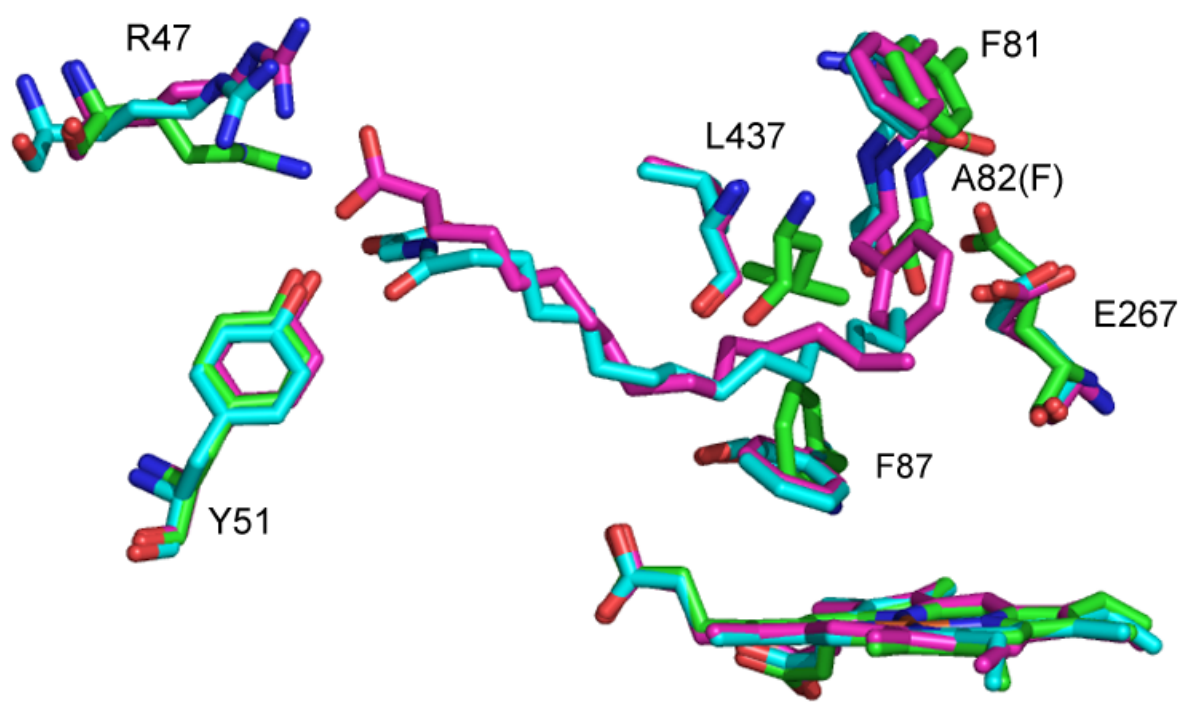

Figure 5
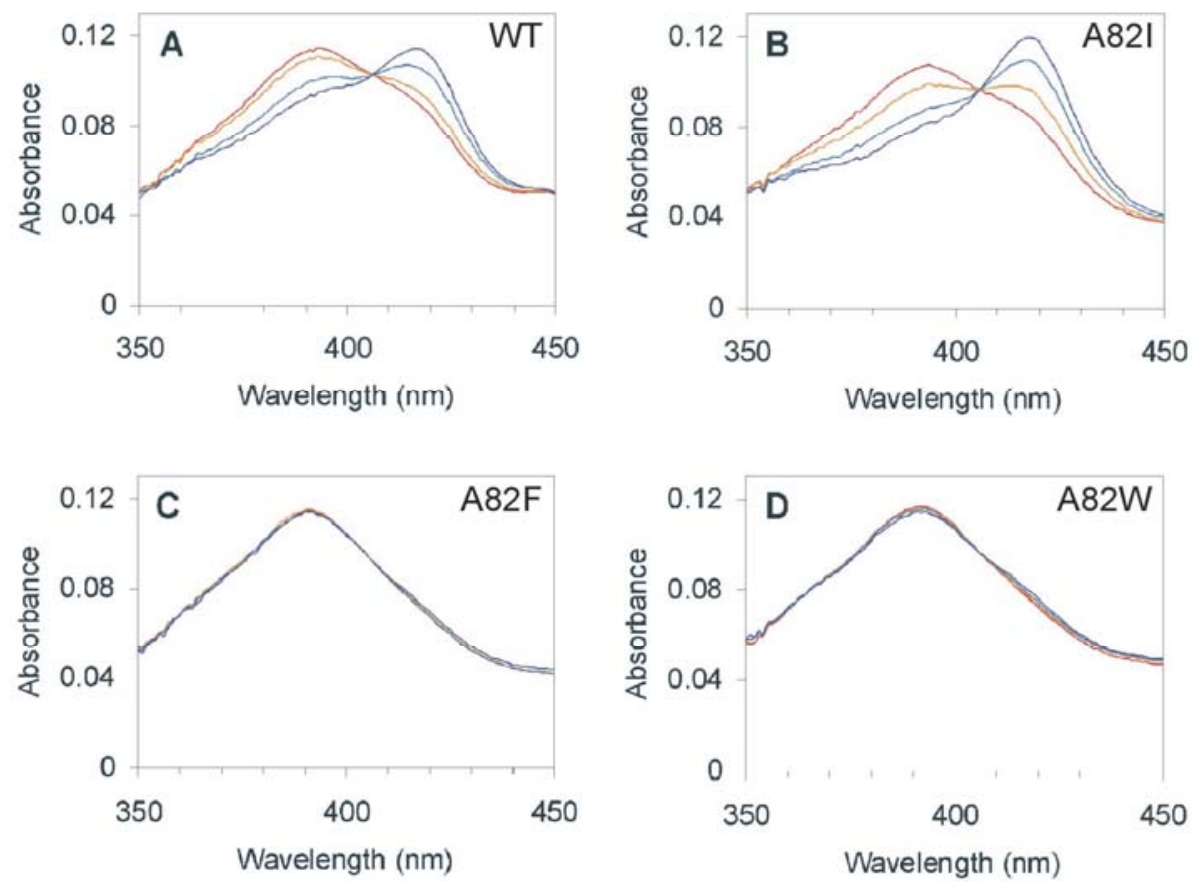
Figure 6
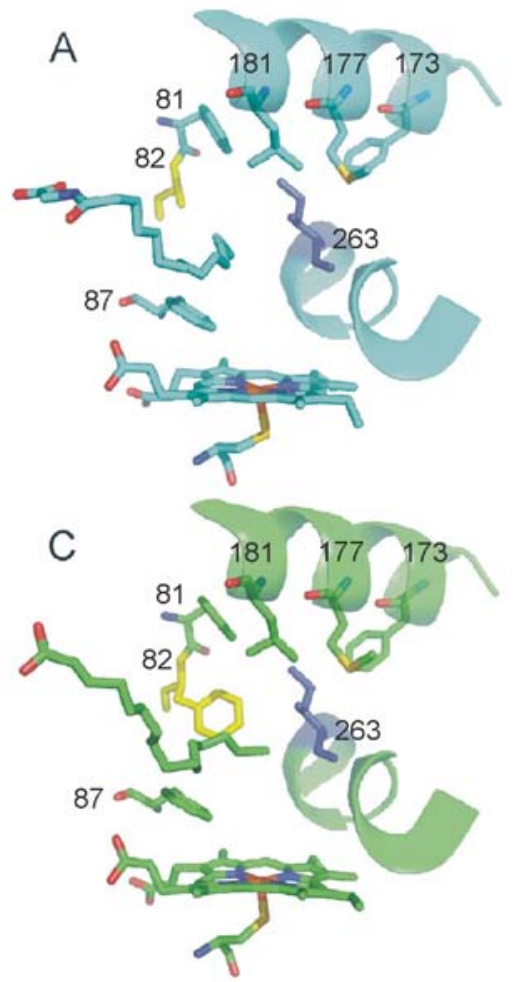
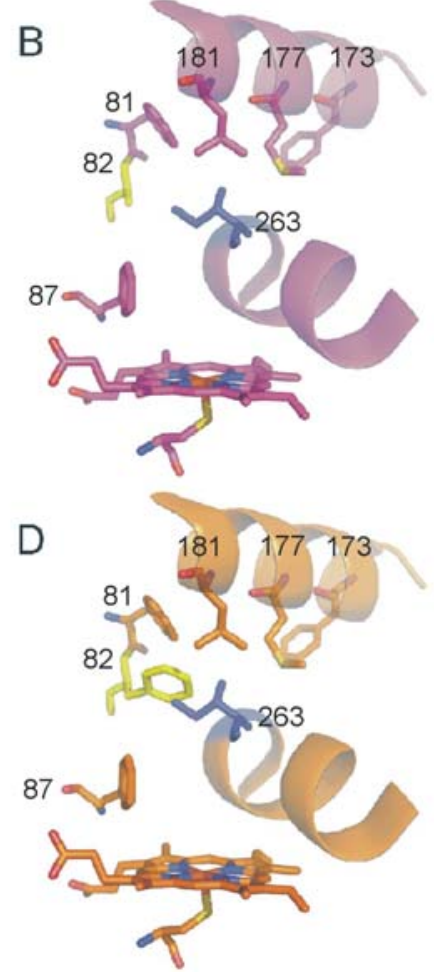

Figure 7
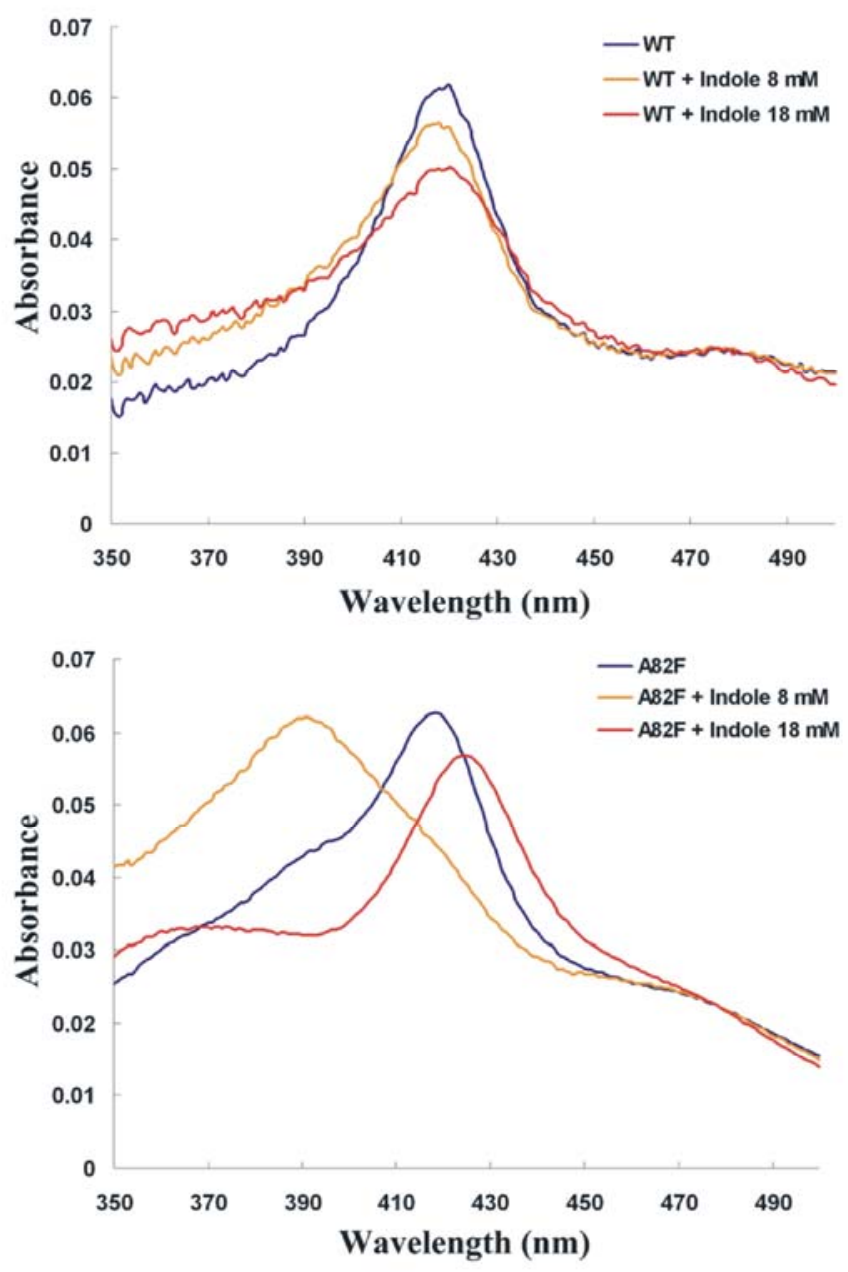
Figure 8
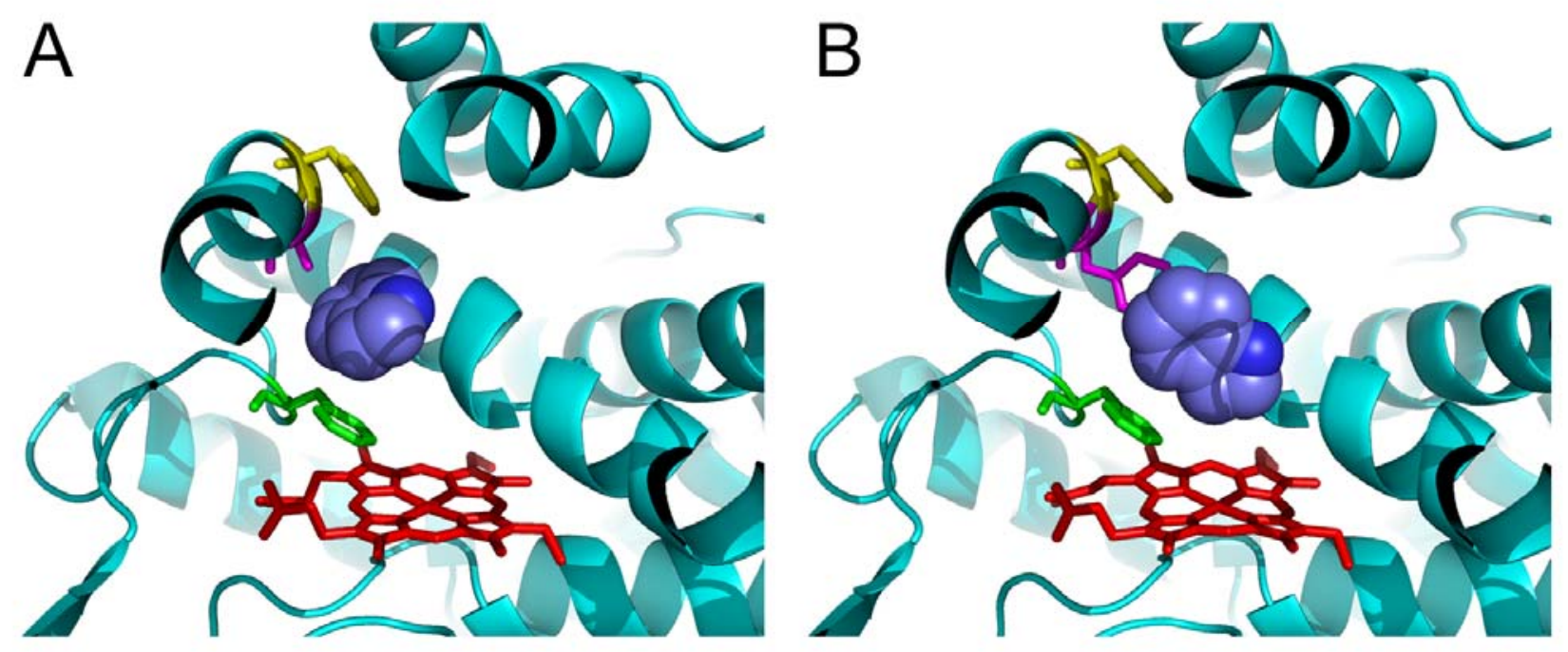


\section{SUPPLEMENTARY INFORMATION}

\section{EXPRESSION CONSTRUCT}

The construct used for expression of P450BM3 in this work was based on the plasmid pGLWBM3 and contained the following modifications, introducing unique restriction sites which allow straightforward recombination of mutations in different regions of the gene:

\begin{tabular}{|l|l|l|}
\hline Nucleotide substitutions & Restriction site & Amino Acid position \\
\hline A4C & NcoI & T1A \\
\hline C768G, T771C & KpnI & R255-Y266 \\
\hline A888T & XhoI & A295-R296 \\
\hline A1242C, T1245A & AvrII & V413-G415 \\
\hline A1413T, C1416A & BstBI & V470-K472 \\
\hline
\end{tabular}

The vector sequence was modified to incorporate a second BstBI site followed by a termination sequence after the end of the P450BM3 gene, to allow generation of P450 domain expression constructs for mutants of interest simply by BstBI digest followed by religation. The modification to the vector sequence was as follows:

CCATGG . . [BM3 gene ] . GGATCCTTTCGAAAATAATTAATTAAGATCC

NCOI

BamHI BstBI Stop

Existing BamHI site destroyed 


\section{The Palmitate Complex OF THE HaEM Domain OF THE A82F MUtANT OF P450 BM3}

Electron density maps calculated using the omit procedure (2 cycles) in SFCHECK (CCP4) showing the substrate binding site for all the six chains in the asymmetric unit.

\section{Chain A}

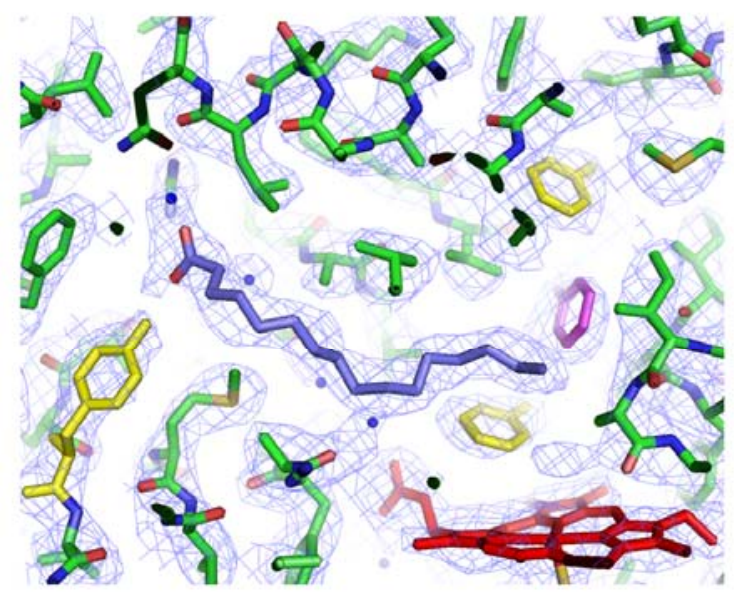

\section{Chain B}

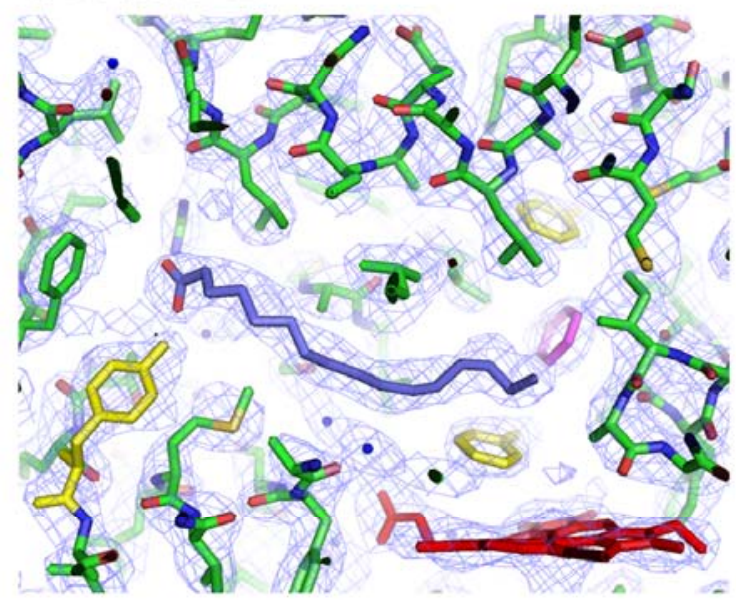

\section{Chain C}

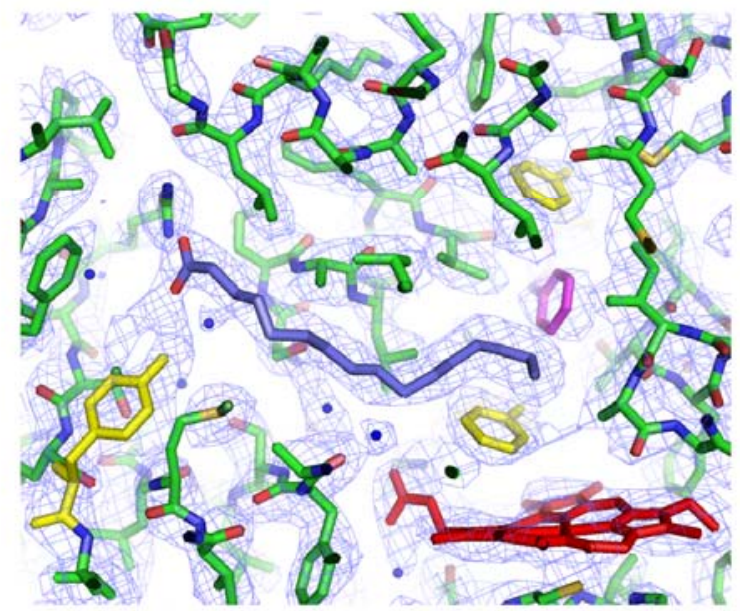

\section{Chain D}

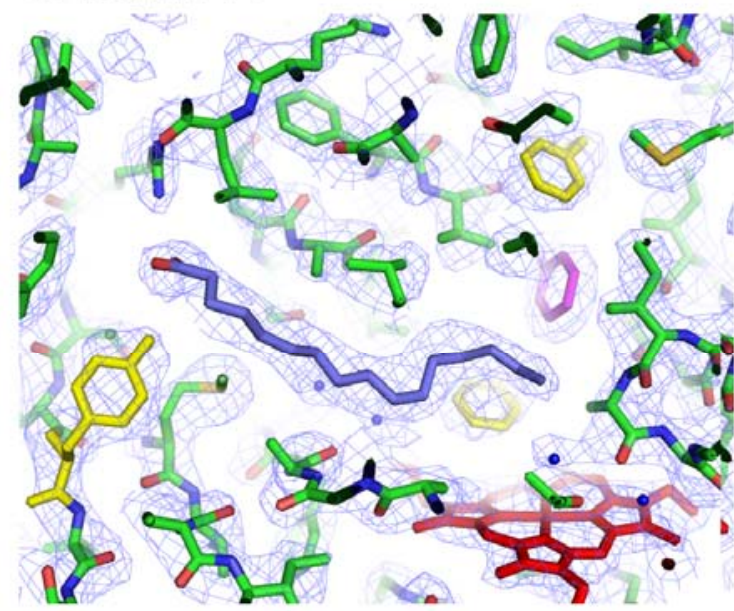

\section{Chain E}

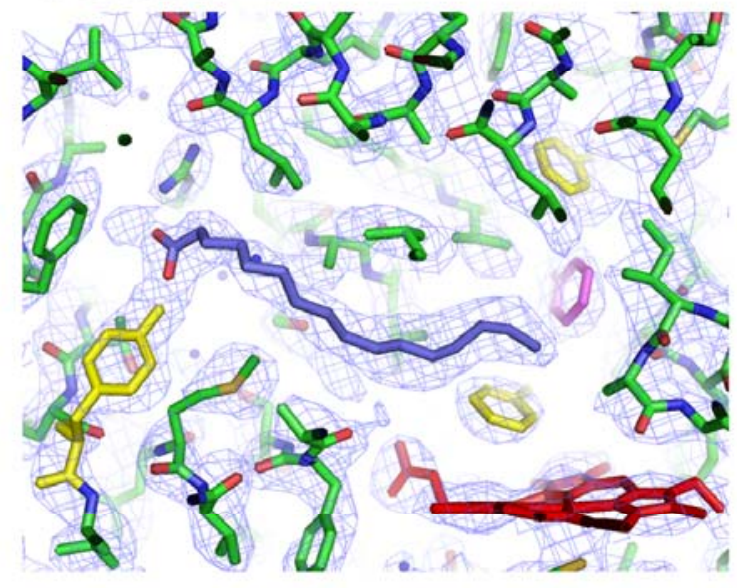

\section{Chain $\mathrm{F}$}

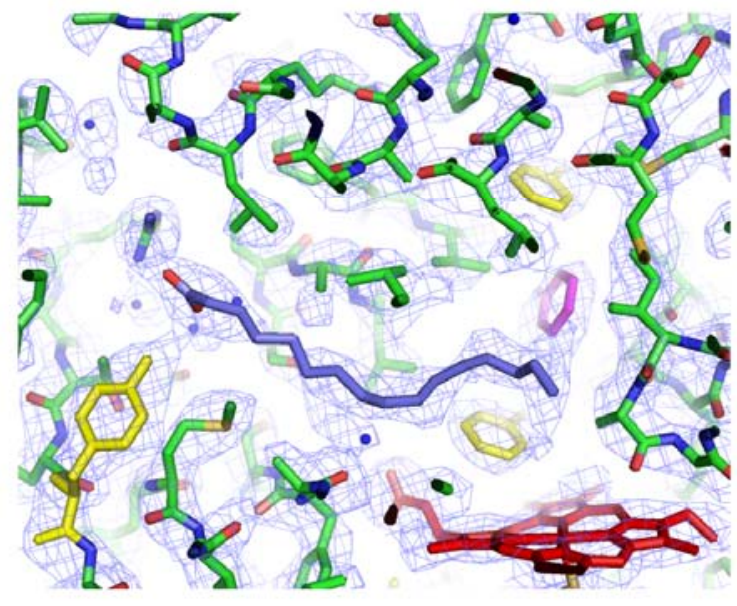




\section{INDOLE METABOLISM}

\subsection{Scheme - Metabolism of indole by cytochromes P450}

Enzyme-catalysed steps are indicated by 'P450', steps which require oxygen but which are not enzymecatalysed by $[\mathrm{O}]$.

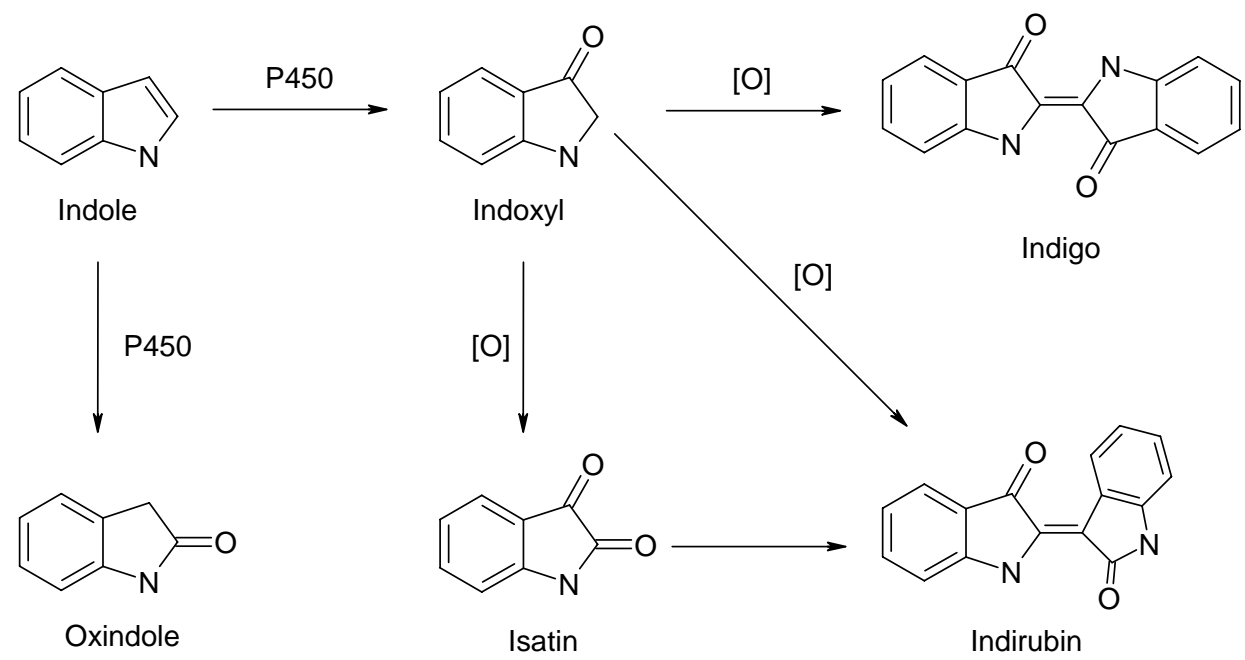

3.2 Analysis of the products of the action of mutants of cytochrome P450 BM3 on indole:

\subsubsection{HPLC of water soluble products}

(A)

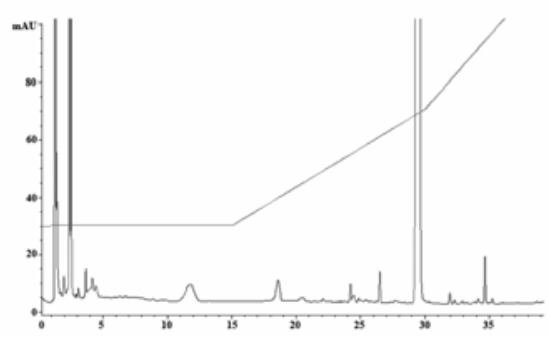

(C)

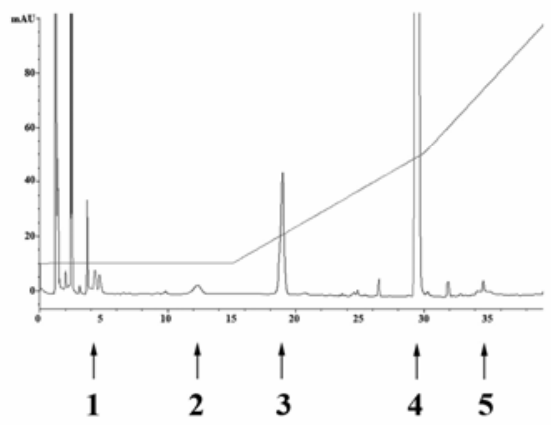

(B)

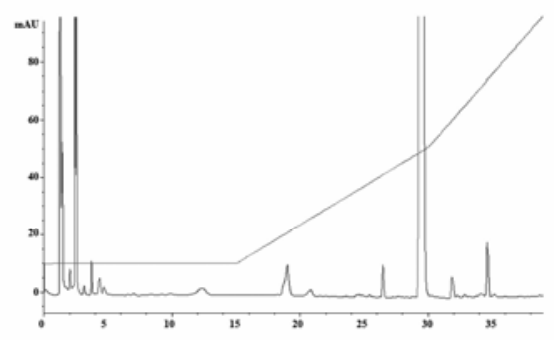

(D)

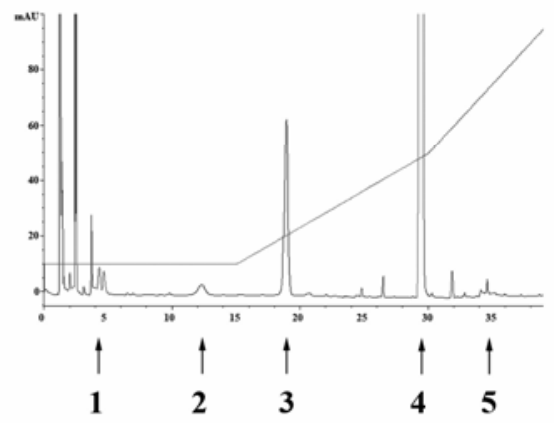

The water-soluble products of indole hydroxylation by P450BM3 wild type (A) and the A82I (B), A82F (C), and A82W (D) mutants analysed using HPLC, measuring absorbance at $240 \mathrm{~nm}$. The retention times of dioxindole (1), isatin (2), oxindole (3), indole (4), and indigo (5) are indicated. 


\subsubsection{Analysis of water-insoluble products re-dissolved in DMSO}

(i) Thin-layer chromatography of the products of indole hydroxylation by wild type P450 BM3 (A), and the $\mathrm{A} 82 \mathrm{~W}(\mathrm{~B}), \mathrm{A} 82 \mathrm{~F}(\mathrm{C})$ or A82I (D) mutants. The migration position of indigo is indicated.

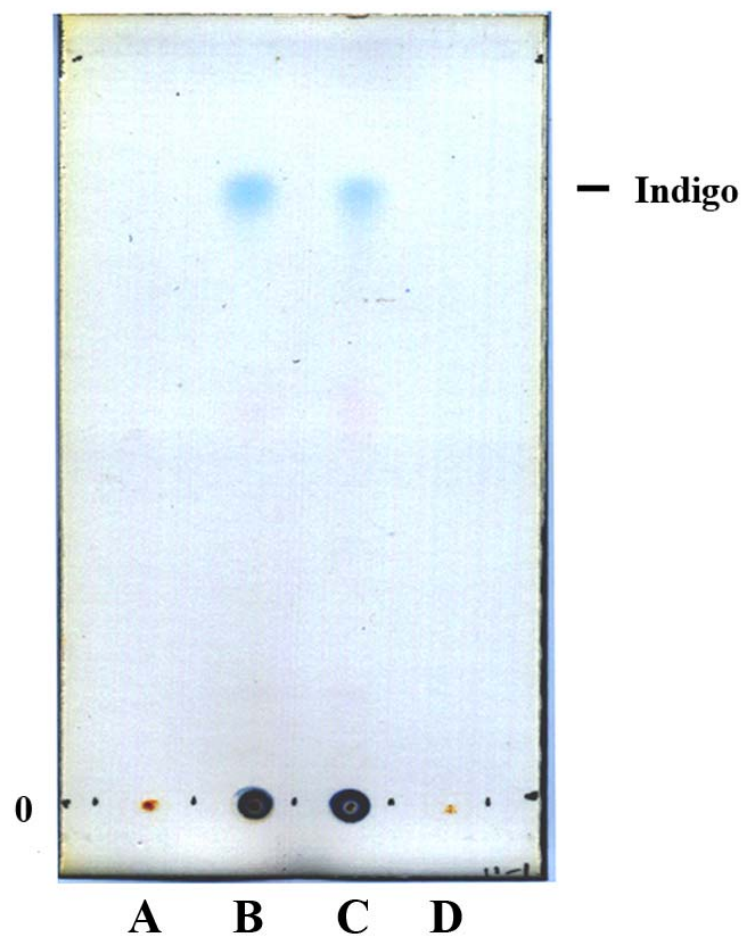

(ii) Optical absorption spectra of indole (green), the product of indole hydroxylation by P450 BM3 A82F (red) and indigo standard (blue).

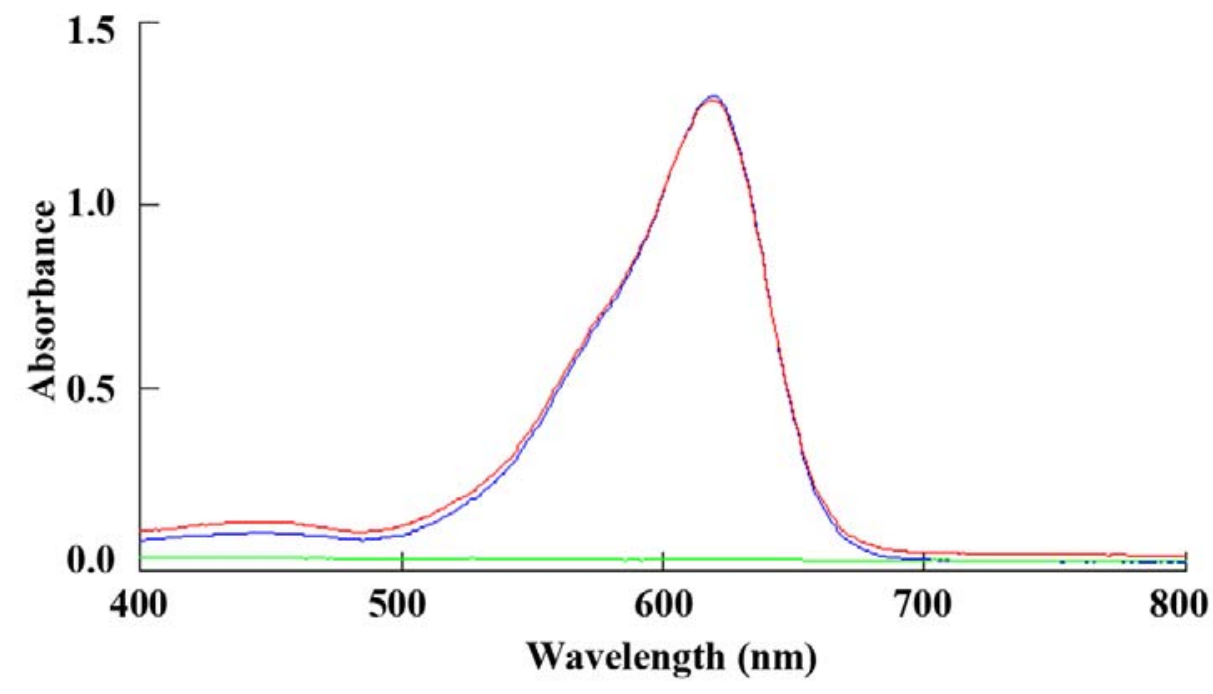


(iii) ${ }^{1} \mathrm{H}$ NMR spectrum of the insoluble product of indole hydroxylation by P450 BM3 A82F. The chemical shifts of 7.622, 7.522, 7.341, 6.958 ppm, correspond to those reported for indigo ( Li, Q. S., Schwaneberg, U., Fischer, P. \& Schmid, R. D. (2000). Directed evolution of the fatty-acid hydroxylase P450 BM-3 into an indole-hydroxylating catalyst. Chem. Eur. J. 6, 1531-1536).

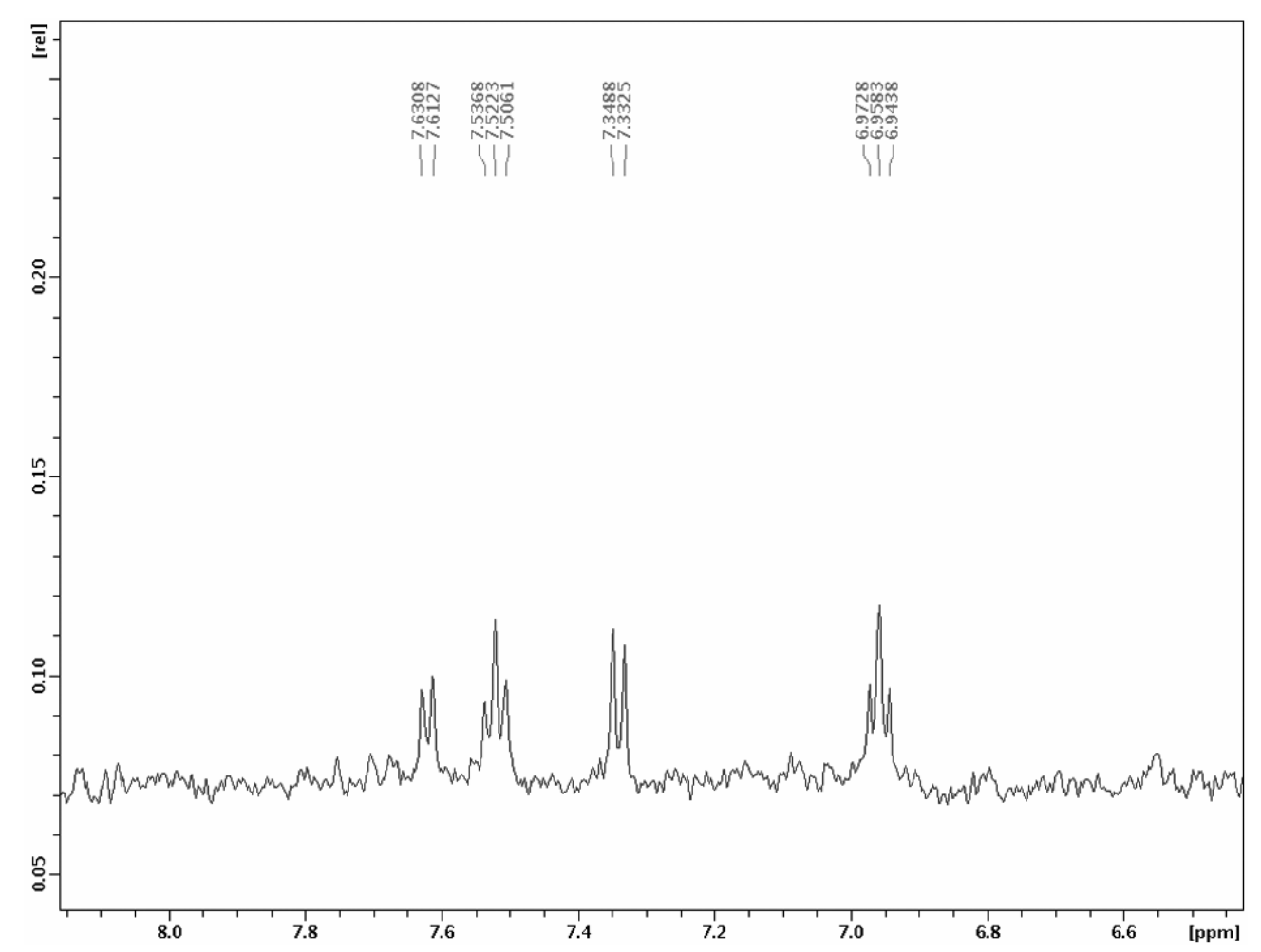

